\title{
Ai-IncRNA EGOT enhancing autophagy sensitizes paclitaxel cytotoxicity via upregulation of ITPR1 expression by RNA- RNA and RNA-protein interactions in human cancer
}

Shouping Xu ${ }^{1 \dagger}$, Peiyuan Wang ${ }^{1 \dagger}$, Jian Zhang ${ }^{1}$, Hao Wu', Shiyao Sui ${ }^{1}$, Jinfeng Zhang ${ }^{1}$, Qin Wang ${ }^{1}$, Kun Qiao ${ }^{1}$, Weiwei Yang ${ }^{2}$, Hongbiao $\mathrm{Xu}^{1}$ and Da Pang ${ }^{1,3^{*}}$ (D)

\begin{abstract}
Background: The biology function of antisense intronic long noncoding RNA (Ai-IncRNA) is still unknown. Meanwhile, cancer patients with paclitaxel resistance have limited therapeutic options in the clinic. However, the potential involvement of Ai-IncRNA in paclitaxel sensitivity remains unclear in human cancer.

Methods: Whole transcriptome sequencing of 33 breast specimens was performed to identify Ai-IncRNA EGOT. Next, the role of EGOT in regulation of paclitaxel sensitivity was investigated. Moreover, the mechanism of EGOT enhancing autophagy sensitizes paclitaxel cytotoxicity via upregulation of ITPR1 expression by RNA-RNA and RNA-protein interactions was investigated in detail. Furthermore, upstream transcriptional regulation of EGOT expression was also investigated by co-immunoprecipitation and chromatin immunoprecipitation. Finally, clinical breast specimens in our cohort, TCGA and ICGC were applied to validate the role of EGOT in enhancing of paclitaxel sensitivity.
\end{abstract}

Results: EGOT enhances autophagosome accumulation via the up-regulation of ITPR1 expression, thereby sensitizing cells to paclitaxel toxicity. Mechanistically, on one hand, EGOT upregulates ITPR1 levels via formation of a pre-ITPR1/ EGOT dsRNA that induces pre-ITPR1 accumulation to increase ITPR1 protein expression in cis. On the other hand, EGOT recruits hnRNPH1 to enhance the alternative splicing of pre-ITPR1 in trans via two binding motifs in EGOT segment 2 (324-645 nucleotides) in exon 1. Moreover, EGOT is transcriptionally regulated by stress conditions. Finally, EGOT expression enhances paclitaxel sensitivity via assessment of cancer specimens.

Conclusions: These findings broaden comprehensive understanding of the biology function of Ai-IncRNAs. Proper regulation of EGOT may be a novel synergistic strategy for enhancing paclitaxel sensitivity in cancer therapy.

Keywords: Ai-IncRNA, EGOT, ITPR1, Paclitaxel, Autophagy, Cancer

\footnotetext{
* Correspondence: pangda@ems.hrbmu.edu.cn

† Shouping Xu and Peiyuan Wang contributed equally to this work.

'Department of Breast Surgery, Harbin Medical University Cancer Hospital,

150 Haping Road, Harbin 150081, China

${ }^{3}$ Heilongjiang Academy of Medical Sciences, 157 Baojian Road, Harbin

150086, China

Full list of author information is available at the end of the article
}

(c) The Author(s). 2019 Open Access This article is distributed under the terms of the Creative Commons Attribution 4.0 International License (http://creativecommons.org/licenses/by/4.0/), which permits unrestricted use, distribution, and reproduction in any medium, provided you give appropriate credit to the original author(s) and the source, provide a link to the Creative Commons license, and indicate if changes were made. The Creative Commons Public Domain Dedication waiver (http://creativecommons.org/publicdomain/zero/1.0/) applies to the data made available in this article, unless otherwise stated. 


\section{Background}

The microtubule-disrupting agent paclitaxel, a plant alkaloid developed from the bark of the Pacific yew tree, Taxus brevifolia, is a first-line chemotherapeutic agent for solid tumors, such as lung, ovarian and breast cancer, and contributes to substantial improvement in patient survival [1]. However, clinical responses to paclitaxel have shown variable sensitivity in cancer patients [2]. Thus, elucidation of the underlying mechanisms of paclitaxel sensitivity and identification of reliable biomarkers that can predict the response to paclitaxel in human cancer are urgently required.

Autophagy is a homeostatic process for degrading cellular materials under cellular stress [3]. Accumulating evidence has shown that autophagy is involved in modulating paclitaxel sensitivity. Tumors expressing diminished levels of autophagy-initiating genes are resistant to paclitaxel therapy, whereas induction of autophagy improves the antitumoral efficiency of the microtubule-disrupting agent paclitaxel $[4,5]$. Furthermore, several studies have proposed that autophagy-related genes/proteins could be investigated as possible prognostic or predictive markers of paclitaxel efficacy in the clinic [6]. However, it is not clear which autophagy-initiating genes can be used as a predictive factor for paclitaxel response.

Long noncoding RNAs (lncRNAs) are a heterogeneous class of transcripts with a minimum length of 200 bases and limited protein-coding potential [7]. LncRNAs exhibit a wide range of expression levels and distinct cellular localizations and are thus a large and diverse class of regulators [8]. LncRNAs can function in cis to regulate the expression of neighboring genes or in trans to perform many roles in various modes [9]. The landscape of lncRNAs dysregulated in autophagy and their molecular mechanisms in autophagy regulatory networks were summarized in our previous study in detail [10]. Studies have demonstrated that lncRNAs such as NBR2 and $A P F$ can regulate autophagy processes with the aid of certain important proteins [10]. Several lncRNAs have also recently been implicated in the modulation of drug resistance [11]. However, the relationship between IncRNA expression and paclitaxel insensitivity caused by abnormal autophagy remains largely unexplored.

Eosinophil granule ontogeny transcript (EGOT), as an antisense intronic long noncoding RNA (Ai-lncRNA), is expressed from ITPR1, which is a ligand-gated ion channel that mediates calcium release from intracellular stores [12, 13]. The biology function of Ai-lncRNAs including EGOT is still largely unknown. In our previous study, we first reported that downregulation of EGOT expression was correlated with advanced malignant status and worse prognosis in breast cancer [14]. Here, we describe a previously unrecognized role of EGOT in increasing sensitivity to paclitaxel via triggering autophagy.
Mechanistically, EGOT enhances autophagosome accumulation via the upregulation of ITPR1 expression in cis and in trans. On one hand, EGOT upregulates ITPR1 levels via formation of a pre-ITPR1/EGOT dsRNA that induces pre-ITPR1 accumulation to increase ITPR1 protein expression in cis. On the other hand, EGOT recruits hnRNPH1 to enhance the alternative splicing of pre-ITPR1 in trans via two binding motifs in EGOT segment 2 (324645 nucleotides) in exon 1. We also uncover that hypoxia induces $E G O T$ transcriptional expression, while estrogen suppresses its expression directly. Finally, EGOT is confirmed to be associated with a favorable prognosis and to enhance paclitaxel sensitivity in breast cancer patients and other human malignancies in our breast cancer cohort and public data cohorts. Given its significance in the autophagy signaling pathway, EGOT may be act as a promising predictive biomarker for paclitaxel response, and proper regulation of $E G O T$ may be a novel synergistic strategy for enhancing paclitaxel sensitivity in human cancer.

\section{Materials and methods}

Breast specimens and clinical assessments

Eligible patients with a histological diagnosis of breast cancer who had received neither chemotherapy nor radiotherapy before surgical resection were recruited to the present study. In total, 258 breast cancer tissues and 258 normal tissues, along with 15 adjuvant chemotherapy regimens, were obtained from Harbin Medical University Cancer Hospital/Center in Harbin, China. All samples were frozen in liquid nitrogen immediately after surgical resection, and only tumors with $>80 \%$ tumor cells were selected for RNA extraction. Two independent senior pathologists confirmed the pathological diagnosis and molecular subtype of each cancer tissue. This study conformed to the clinical research guidelines and was approved by the research ethics committee of Harbin Medical University Cancer Hospital. We obtained written informed consent from all patients. For 184 patients, telephone follow-up was performed to verify survival status after primary treatment. Among them, 159 patients received paclitaxel treatment.

\section{Cell culture and treatments}

The human cervical cancer cell line HeLa and 10 breast cancer cell lines (MCF7, T47D, UACC-812, SK-BR-3, MDA-MB-453, MDA-MB-231, Hs578T, HCC70, BT549, and MDA-MB-468) were obtained from the Chinese Academy of Sciences Cell Bank and Cellbio (China), respectively. All cell lines were periodically authenticated (Cellbio). Unless specifically indicated, cells were cultured in DMEM (R10-017-CV, Corning, USA), RPMI-1640 (R10-040-CM, Corning) or Leibovitz's L15 (PYG0038, Boster, China) medium supplemented with $10 \%$ fetal bovine serum (0500, ScienCell, USA) at $37^{\circ} \mathrm{C}$ with $5 \% \mathrm{CO}_{2}$ or air and $95 \%$ humidity. In estrogen-related experiments, 
MCF7 and T47D cells were washed three times with phenol red-free DMEM and subjected to hormone deprivation for up to 3 days with $10 \%$ activated charcoal-absorbed fetal calf serum (FCS; P30-2302, PAN) before proceeding to the next steps. The reagents used were $17 \beta$-oestradiol (E8875, Sigma, USA), the estrogen receptor antagonist tamoxifen (T5648, Sigma) and ICI 182780 (Asc-131, Ascent Scientific, USA). Autophagosome and LC3 experiments were performed as previously described [15] and were performed in one of two ways. Cells $\left(10-20 \times 10^{4}\right.$ cells $\left./ \mathrm{ml}\right)$ were plated in medium containing $10 \%$ serum. After $24 \mathrm{~h}$, the medium was changed to that containing $0.1 \%$ serum, and the cells were collected $48 \mathrm{~h}$ later with or without $100 \mathrm{mM}$ CQ (ab142116, Abcam, USA) treatment for $1 \mathrm{~h}$ before collection. Alternatively, the cells were cultured with EBSS (E2888, Sigma) for the indicated durations before collection. For the ubiquitination assay, HeLa cells were treated with MG132 $(10 \mu \mathrm{M} / \mathrm{l})$ (ab141003, Abcam) for $40 \mathrm{~min}$. Protein levels were detected by immunoblotting and quantified by densitometry. For the CHX chase assay, HeLa cells were incubated with $50 \mu \mathrm{g} / \mathrm{ml}$ CHX (Catalogue Number HY-12320, MCE, USA) for the indicated durations $(0,15,30,60,120$, and $180 \mathrm{~min}$ ) as previously described [16]. For transcriptional inhibition experiments, actinomycin D $(6 \mu \mathrm{g} / \mathrm{ml})$ (A1410, Sigma) was added to the cells, and samples were harvested at the indicated time points $(0,0.5,1,2,4$, and $8 \mathrm{~h})$ as previously described [17].

RNA extraction, reverse transcription and quantitative RTPCR and PCR array

Total RNA and miRNA were extracted from cells using the E.Z.N.A. Total RNA Kit I (Catalogue Number R6834-01, Omega Bio-Tek, USA). First-strand cDNA was prepared with the Transcriptor First Strand cDNA Synthesis Kit (Catalogue Number 04897030001, Roche, USA). Real-time PCR was performed using FastStart Universal SYBR Green Master (ROX) (Catalogue Number 04913914001, Roche) on a 7500 Fast Real-Time PCR system (ABI, USA). For quantification of gene expression, we used the $2^{-\triangle \Delta C t}$ method. GAPDH expression was used for normalization. The primer sequences were synthesized by Shanghai Generary Biotech Co., Ltd. and are included in Additional file 1: Table S1.

\section{Lentivirus production and infection}

Recombinant lentiviruses expressing the lncRNA EGOT, shEGOT, shITPR1, shESR, EGOT-MS2, Flag-MCP2 and controls were constructed by Sangon Biotech Company (China). Concentrated viruses were used to infect $5 \times 10^{5}$ cells in a 6-well plate with $4-6 \mu \mathrm{g} / \mathrm{ml}$ polybrene $(107,689$, Sigma). The infected cells were then subjected to selection with $1 \mu \mathrm{g} / \mathrm{ml}$ puromycin (Catalogue Number 540411, Calbiochem, USA) for two weeks. Stable overexpression cell lines or knockdown cell lines were identified using qRT-PCR or western blotting. The shRNA sequences are provided in Additional file 1: Table S1.

\section{Co-immunoprecipitation, western blot assay and antibodies} Co-immunoprecipitation assays were carried out by using the Pierce $^{\mathrm{Tm}}$ Crosslink Magnetic IP/Co-IP Kit (Catalogue Number 88805, Thermo Fisher, USA) according to the manufacturer's protocol. Western blotting was performed according to the previously described procedures [18]. Anti-LC3B (ab48394, 1:1000), anti-IP3R (ab5804, 1:1000) antibody, anti-SQSTM1/P62 (ab91526, 1:1000) antibody, anti-hnRNPH1 (ab10374, 1:1000) antibody, anti-HMGB1 (ab79823, 1:1000) antibody, anti-RIP140 (ab42126,1:500) antibody, goat anti-rabbit IgG H\&L (HRP) (ab6721, 1:10,000), and goat anti-mouse IgG H\&L (HRP) (ab6789, 1:10,000) were obtained from Abcam. Anti-tubulin (sc-73,242, 1:1000) antibody was purchased from Santa Cruz Biotechnology. Anti-estrogen receptor (WL00940, 1:1000) antibody and anti-GAPDH (WL01114, 1:1000) antibody were obtained from Wanleibio (China). Anti-Bax (D2E11, 1:1000) (5023) antibody, anti-Bcl2 (2872, 1:1000) antibody and c-Jun (60A8) Rabbit mAb (\#9165, 1:500) antibody were obtained from Cell Signaling Technologies (CST). Total cell lysates were prepared using a $1 \times$ sodium dodecyl sulfate buffer. Identical quantities of proteins were separated by sodium dodecyl sulfate-polyacrylamide gel electrophoresis and transferred onto nitrocellulose filter membranes. After incubation with specific antibodies, the blots were incubated with goat anti-rabbit IgG H\&L (HRP) and goat anti-mouse IgG H\&L (HRP) for $1 \mathrm{~h}$ at room temperature. The proteins were detected using a FluorChem HD2 (Protein Simple, USA).

\section{Tandem mRFP-GFP fluorescence microscopy}

Tandem monomeric RFP-GFP-tagged LC3 (tfLC3) (HBAP2100001, Hanbio, China) was used to monitor autophagic flux as previously reported. LC3-II relocalized to the autophagosomal membranes during autophagy. Thus, the accumulation of mRFP-GFP-LC3 puncta is an effective way to detect autophagosomes. When tfLC3 is located in autolysosomes, this form of LC3 displays only red fluorescence since the GFP signal is sensitive to the acidic condition in the lysosome lumen, whereas the RFP signal is more stable. To evaluate tandem fluorescent LC3 puncta, $48 \mathrm{~h}$ after tfLC3 transfection, cells were washed once with $1 \times$ PBS, incubated with EBSS (E2888, Sigma) for the indicated durations and then directly sent out for confocal microscopy analysis. Images of samples were captured using a Zeiss LSM 710 confocal microscope system (Carl Zeiss, Germany) and processed with ZEN LE software (Carl Zeiss). 


\section{Subcellular fractionation}

Nuclear/cytoplasmic isolation was carried out by using the NE-PER ${ }^{\mathrm{m}}$ Nuclear and Cytoplasmic Extraction Reagents (Catalogue Number 78835, Thermo Fisher) according to the manufacturer's protocol. Subcellular fractions were prepared as follows. Cytoplasmic and nuclear fractions were divided for RNA extraction. GAPDH and $U 1$ were used as qRT-PCR markers of cytoplasmic and nuclear RNAs, respectively.

\section{Transmission electron microscopy}

Conventional electron microscopy was performed as previously described [19]. In brief, cells were fixed with $2.5 \%$ glutaraldehyde and then postfixed with $1 \%$ osmium tetroxide, dehydrated in a graded ethanol series and embedded in Embed 812 resin. Ultrathin sections were mounted on copper grids and then double-stained with uranyl acetate and lead citrate. The samples were examined and photographed with an FEI Tecnai spirit transmission electron microscope.

\section{RNA-FISH assay}

To detect EGOT and pre-ITPR1 mRNAs, we used the QuantiGene $^{\circ}$ ViewRNA ISH Cell Assay Kit (Catalogue Number QVC0001, Thermo Fisher) to perform the QuantiGene ViewRNA FISH assay according to the manufacturer's protocol. EGOT, ITPR1 and ACTB (as control) hybridization was carried out using cy3, cy5, and 488-nm DNA-oligonucleotide probes in a moist chamber, respectively. After digestion with a working protease solution, slides were incubated with RNase III (AM2290, Life Technologies, USA) or RNase A (AM2272, Life Technologies) for $2 \mathrm{~h}$ if RNase enzymatic activity was to be determined. Standard immunofluorescence and imaging were performed by confocal microscopy. The details of the probe sets and corresponding gene sequences are provided in Additional file 1: Table S2.

\section{TUNEL assay}

To detect apoptosis in sections of tumor tissues, TUNEL assay was performed according to the manufacturer's instructions (Catalogue Number 11684795910, Roche) as previously described [20]. The sections were analyzed by fluorescence microscopy (Olympus, Japan).

\section{RNA pull-down assay}

The Flag-MS2bp-MS2bs-based RNA pull-down assay was carried out by using the Anti-Flag M2 Affinity Gel (Catalogue Number A2220, Sigma) as previously described [21]. In short, lentivirus Flag-MS2bp and lentivirus EGOT MS2bs were cotransfected into breast cancer cells, and the cells were harvested after $48 \mathrm{~h}$. Approximately $1 \times 10^{7}$ cells were lysed in soft lysis buffer $(20 \mathrm{mM}$ Tris-Cl, $\mathrm{pH} 8.0,10 \mathrm{mM} \mathrm{NaCl}, 1 \mathrm{mM}$ EDTA, and 0.5\% NP-40) containing RNasin (80 units $/ \mathrm{ml}$ ). Fifty microliters of Anti-Flag M2 Magnetic beads was added to each binding reaction tube and incubated at $4{ }^{\circ} \mathrm{C}$ overnight. The beads were washed three times with lysis buffer and boiled in $1 \mathrm{x}$ loading buffer for $10 \mathrm{~min}$. Finally, the retrieved proteins were analyzed by a NanoLC-ESI-MS/ MS system (ProtTech, China).

\section{Chromatin immunoprecipitation (ChIP) and ChIP-seq}

Analysis of genome-wide NRIP1 and AP-1 occupancy was carried out using specific and internally validated antibodies. We purchased the EZ-ChIP ${ }^{\mathrm{Tx}}$ Chromatin Immunoprecipitation Kit (Catalogue Number \#17371, Millipore, USA) to perform ChIP and ChIP-seq using MCF7 cells as previously described [22]. Briefly, MCF7 cells were subjected to hormone deprivation for up to 3 days and then treated with $1 \mathrm{nM}$ E2 or ethanol (control) for $6 \mathrm{~h}$. Approximately $2 \times 10^{7}$ cells were used for each ChIP or ChIP-Seq assay. Chromatin DNA precipitated by polyclonal antibodies against AP-1 or NRIP1 was purified with the Universal DNA purification kit (DP214, Tiangen, China) according to the manufacturer's protocol. Rabbit anti-RIP140 (ab42126, Abcam) and rabbit anti-c-JUN (60A8) (Catalogue Number \#9165, CST) antibodies and rabbit IgG (sc-2027, Santa Cruz, USA) were used. ChIP-PCR enrichment of target loci was normalized to input DNA and reported as \% input \pm s.e.m. ChIP libraries were prepared using ChIP DNA according to the BGISEQ-500ChIP-Seq library preparation protocol. In-depth whole-genome DNA sequencing was performed by BGI (Shenzhen, China). ChIP-seq data were deposited in the NCBI SRA: SRP149488 (https://www. ncbi.nlm.nih.gov/sra/SRP149488).

\section{Transient transfection of cells}

For downregulation of NRIP1 and hnRNPH1 expression, siRNAs targeting NRIP1 and hnRNPH1 were purchased from Sigma. Cancer cells were transfected with $100 \mathrm{pM}$ siRNA using Lipofectamine 2000 (11668-019, Invitrogen, USA). The siRNA sequences are provided in Additional file 1: Table S1. After HeLa and T47D cells $\left(5 \times 10^{5}\right.$ cells/ well) were cultured in 6-well plates for $24 \mathrm{~h}$, siRNA or the corresponding controls were transfected into the cells by Lipofectamine 2000 reagent in accordance with the manufacturer's protocol. Total RNA was extracted $48 \mathrm{~h}$ after transfection.

\section{Animal experiment}

Female athymic BALB/c nude mice (5 weeks old) were obtained from Beijing Vital River Laboratory Animal Technology Co, Ltd. (Beijing, China). All of the animal experiments were performed according to approved protocols and in accordance with the guidelines of the Guide for the Care and Use of Laboratory Animals 
(Institute of Laboratory Animal Resources, Commission on Life Sciences, National Research Council). The protocol was approved by the Institutional Animal Care and Use Committee of Center of Harbin Medical University. Approximately $8 \times 10^{6}$ UACC-812 cells resuspended in $0.2 \mathrm{ml}$ of $25 \%$ phenol red-free Matrigel (Catalogue Number 356234, Corning) with $0.9 \% \mathrm{NaCl}$ were injected in the axilla of five-week-old BALB/c mice. After the tumors grew to approximately $300-500 \mathrm{~mm}^{3}$ in size, $15 \mathrm{mg} / \mathrm{kg}$ paclitaxel was administered once every 4 days for a total of three courses. Tumor volume was measured once every 2 days by using calipers at the indicated time points. The tumor volume was estimated by the following formula: length $\times$ width $\times$ width $/ 2$. The whole body weight of mice was measured once every 2 days as indicated. All mice were euthanized by intraperitoneal injection of $200 \mathrm{mg} / \mathrm{kg}$ pentobarbital at the end of the experiment.

\section{Immunohistochemistry}

Immunohistochemical (IHC) detection of ITPR1 and LC3B was performed on each slide. Each section was incubated with anti-LC3B (1:200) antibody or anti-IP3R (1:200) antibody solution. The proportion and intensity of ITPR1 and LC3 staining were evaluated in a series of 10 randomly selected high-power fields (magnification, $400 x$ ), which were considered to represent the average expression. The IHC staining intensity was graded as 0 (no staining), 1 (weak staining = light yellow), 2 (moderate staining = yellow brown) or 3 (strong staining= brown). The proportion of positively stained tumor cells in a field was scored as 0 (no positive tumor cells), 1 (fewer than $10 \%$ positive tumor cells), 2 ( $10-50 \%$ positive tumor cells) or 3 (more than $50 \%$ positive tumor cells). The staining index (SI) for each sample was obtained by multiplying the intensity and proportion values, with a score of less than 4 being classified as low expression.

\section{Library preparation for IncRNA sequencing}

A total of $3 \mu \mathrm{g}$ of RNA per sample was used for downstream RNA sample preparation. Ribosomal RNA was removed using the Ribo-Zero ${ }^{\text {ma }}$ Gold kit (Epicentre, Wisconsin, USA). Subsequently, sequencing libraries were generated according to the manufacturer's recommendations, and the libraries were sequenced on an Illumina HiSeq 2500 platform to generate 100 -bp paired-end reads. Raw sequencing and processed RNASeq data from this study have been deposited into the NCBI GEO database under accession number GSE71651 (http://www.ncbi.nlm. nih.gov/geo/query/acc.cgi?token=obcxosaur xoppwx \& acc $=$ GSE71651).

\section{Access and analysis of public data}

GDS723, GDS4121, GDS1453 and GSE11324 were downloaded from GEO datasets (http://www.ncbi.nlm. nih.gov/geo/) and processed according to our previous study [23]. Genome-wide EGOT and ITPR1 expression profiles and clinical pathology information for human cancers were downloaded from The Cancer Genome Atlas (TCGA) (https://tcga-data.nci.nih.gov/), International Cancer Genome Consortium (ICGC) (http://icgc.org/) and Cancer Cell Line Encyclopedia (CCLE) (http://www.broad institute.org/ccle). All transcripts were normalized by $\log _{2}$ transformation. The expression of EGOT or ITPR1 was dichotomized using a study-specific median expression as the cut-off to define high values (at or above the median) versus low values (below the median). Guilt-by-association analysis was performed according to our previous study [23]. Correlations between genes were assessed by Pearson correlation coefficients. Unpaired Student's t-tests were used to detect significant differences among tumors or between tumor and normal samples. The overall survival (OS) and relapse-free survival (RFS) were calculated as the time from surgery until the occurrence of death and relapse, respectively. The log-rank test was used to examine the survival difference between different patient groups. All statistical tests were two-sided, and $P<0.05$ indicated statistical significance.

\section{Statistical analysis}

Data are presented as the mean \pm s.d. of at least three independent experiments for each cellular experimental group and at least five independent experiments for each animal group. Student's t-tests were used to determine statistically significant differences between groups. Correlations between EGOT expression and a pathological response were determined by the chi-square test. All statistical tests were two-sided, and $P<0.05$ indicated statistical significance. Statistical analysis was performed using R.3.4 graphics software and GraphPad Prism software (GraphPad Software, USA).

\section{Results}

\section{Ai-IncRNA EGOT enhances paclitaxel sensitivity in human} cancer

Whole transcriptome sequencing of 33 breast specimens showed that Ai-lncRNA EGOT was expressed at low levels in cancer tissues compared with its levels in adjacent normal tissues (Fig. 1a and Additional file 2: Figure S1a). This result was subsequently validated in 258 pairs of breast cancer tissues and adjacent normal tissues in the Harbin Medical University Cancer Centre (HMUCC) cohort (Fig. 1b) and in data from other cancer contexts in TCGA (Additional file 2: Figure S1b). 


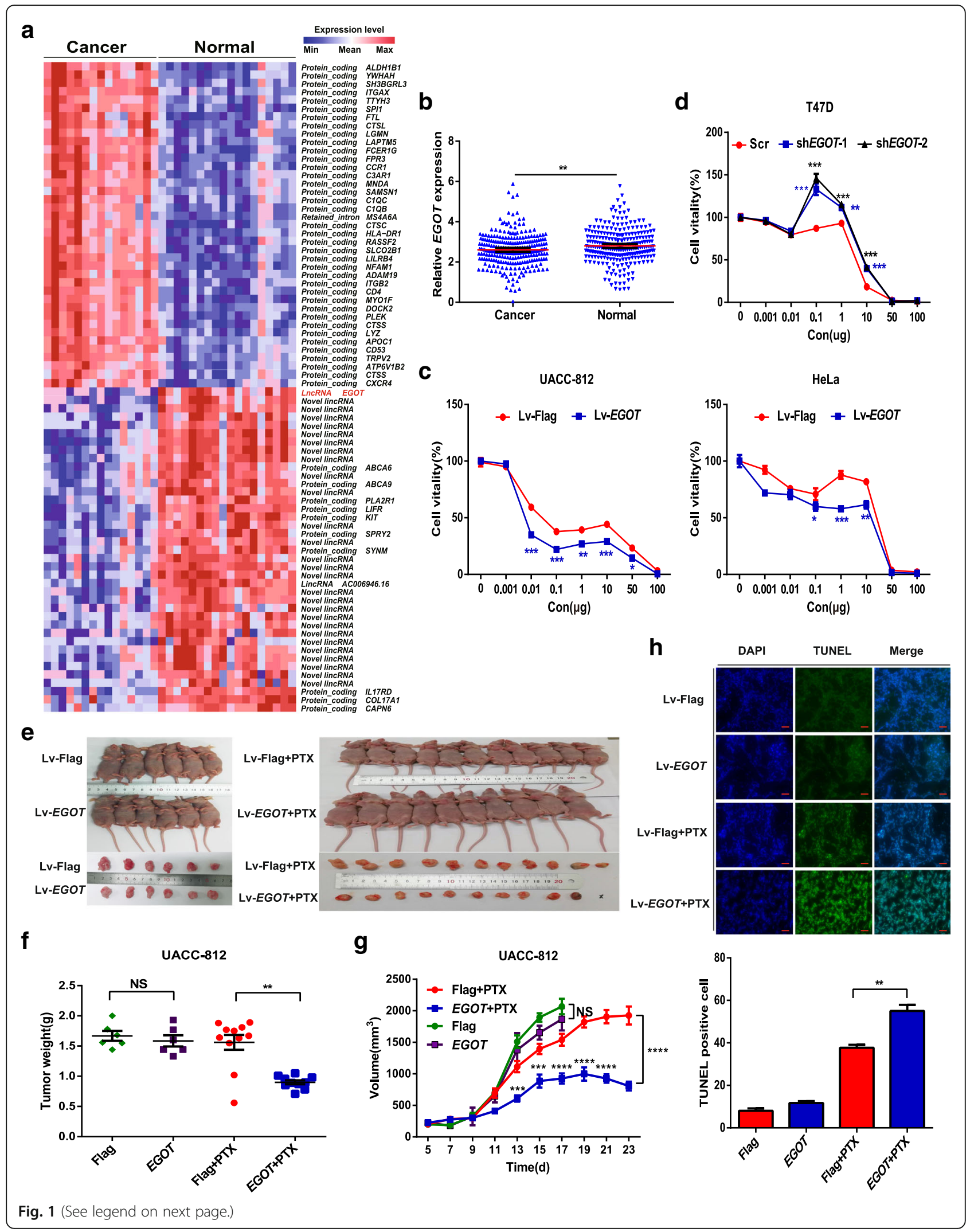


(See figure on previous page.)

Fig. 1 Ai-IncRNA EGOT enhances paclitaxel sensitivity in human cancer. a Heatmap of the whole transcriptomes of 33 breast specimens indicating that InCRNA EGOT expression levels are lower in cancer tissues than in normal tissues. Colors correspond to the expression level indicated by the $\log _{2}$-transformed scale bar below the matrix. Red and blue reflect Max and Min levels, respectively. $\mathbf{b}$ qRT-PCR analysis of EGOT expression in breast cancer tissues and adjacent normal tissues from 258 patients in the HMUCC cohort. GAPDH served as a reference for EGOT. $\mathbf{c}$, d Cell viability was analyzed by CCK-8 assay after paclitaxel treatment for $48 \mathrm{~h}$ in EGOT overexpression and knockdown cells. e Mice and tumours from the UACC-812 Lv-EGOT and control (Lv-Flag) groups with or without treatment of paclitaxel. $\mathbf{f}$ The weight of tumours excised from mice in the UACC-812 LV-EGOT and control groups with or without treatment of paclitaxel. $\mathbf{g}$ The volumes of tumours established in UACC-812 LV-EGOT and control groups with or without $15 \mathrm{mg} / \mathrm{kg}$ paclitaxel once every four days at the indicated time (day 11, day 15 and day 19). $\mathbf{h}$ Representative sections (upper) and average number of TUNEL-positive cells (bottom) in subcutaneous tumors. Scale bar, $50 \mu m$. Data are shown as the mean \pm s.d. Student's t-test was used for statistical analysis: ${ }^{*} P<0.05$; ${ }^{* *} P<0.01$; ${ }^{* *} P<0.001$; and ${ }^{* * *} P<0.0001$. Data represent at least three

independent experiments

To further investigate the biological functions of EGOT, we performed guilt-by-association analysis and found that EGOT was involved in multiple cellular microtubule-associated functions (Additional file 1: Table S3). Because paclitaxel is commonly used as a microtubule-disrupting agent, we suspected that EGOT may impact paclitaxel sensitivity in tumor cells. As expected, analysis of public data from CCLE showed that EGOT increases the sensitivity of tumor cells to paclitaxel (Additional file 2: Figure S1c). To validate the potential function of EGOT in mediating the paclitaxel response, we then generated stable overexpression and knockdown of EGOT in cancer cell lines (Additional file 2: Figure S1d-f). As shown in Fig. 1c and Additional file 2: Figure S1g, we determined that EGOT overexpression significantly increased the sensitivity of cancer cells to paclitaxel. Conversely, silencing EGOT protected cancer cells from paclitaxel (Fig. 1d).

Furthermore, we found that there was no distinct difference of xenografts in the presence of EGOT or control without treatment of paclitaxel in UACC-812 cell xenograft models (Fig. 1e-g). However, EGOT overexpression (Additional file 2: Figure $\mathrm{S} 1 \mathrm{~h}$ ) in UACC-812 and HeLa cell xenografts grew more slowly than control ones when treated with paclitaxel (Fig. 1e and Additional file 2: Figure S1i). Moreover, the weight and volume of the tumors were decreased in the EGOT overexpression group compared with those in the control group with treatment of paclitaxel (Fig. If and g). Similar results were obtained in HeLa cell xenografts (Additional file 2: Figure S1j and k). More importantly, cell apoptosis was much higher in the EGOT overexpression group than in the control group when treated with paclitaxel (Fig. 1h). Collectively, these data demonstrate that EGOT enhances the sensitivity of human cancer cells to paclitaxel both in vitro and in vivo.

\section{EGOT expression is positively correlated with ITPR1 expression}

We then sought to identify interacting partner(s) involved in EGOT-mediated paclitaxel sensitivity. Certain mammalian lncRNAs are embedded in the intronic-antisense regions of protein-coding genes and regulate the genes to exert their function [24]. Thus, we hypothesized the existence of a functional relationship between EGOT and ITPR1. To study this possibility, by analyzing whole-transcriptome sequencing data, we found that $E G O T$ expression was positively correlated with ITPR1 expression at the mRNA level (Additional file 2: Figure S2a). The result was validated using breast cancer specimens in the HMUCC cohort (Fig. 2a); moreover, EGOT expression was also positively correlated with ITPR1 protein expression in the HMUCC cohort (Additional file 2: Figure S2b). To expand the generalizability of our results, human cancer data from 33 cancer contexts, including breast cancer, in TCGA were analyzed, and the results further validated as above (Fig. 2b and c; Additional file 1: Table S4). Furthermore, examination of CCLE data also revealed that EGOT expression was positively correlated with ITPR 1 mRNA expression in human breast cancer cell lines and other cancer cell lines (Fig. 2d and e; Additional file 2: Figure S2c-f). These results indicate that EGOT expression is positively correlated with ITPR1 expression in cancer.

To further validate the relationship between EGOT and ITPR1expression, we detected the genes near the $E G O T$ locus (less than $2 \mathrm{Mb}$ ). EGOT overexpression was shown to increase the expression of ITPR1 mRNA (Fig. $2 \mathrm{f}$ and g), while EGOT knockdown decreased the expression of ITPR1 mRNA (Additional file 2: Figure S2 g). Consistent with these results, ITPR1 protein levels were also increased following EGOT overexpression and were decreased following EGOT knockdown (Fig. 2h). However, $E G O T$ expression did not significantly change following ITPR1 knockdown (Additional file 2: Figure S2 h). Collectively, these data suggest that EGOT may induce ITPR1 expression in human cancer.

\section{EGOT induces autophagy to enhance paclitaxel sensitivity through ITPR1}

To illustrate that ITPR1 plays a functional role in autophagy in human cancer cells, we investigated autophagic activity in cells after $48 \mathrm{~h}$ of growth under low-serum $(0.1 \%)$ conditions or in Earle's balanced salt solution (EBSS) to simulate stress-induced autophagy, as previously described [15]. Knockdown of ITPR1 decreased LC3-II/ 


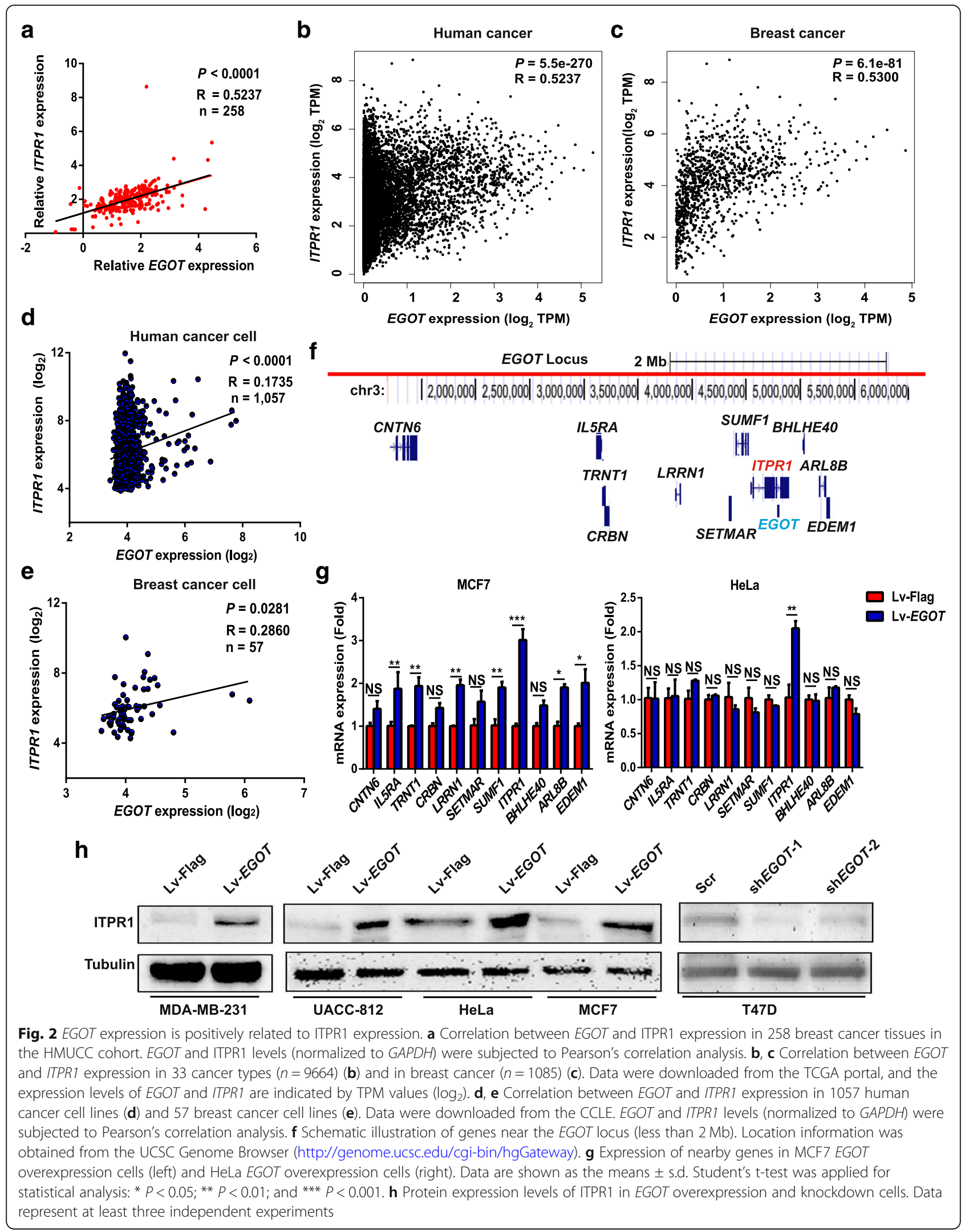


LC3-I levels and increased P62 expression in the presence of chloroquine (CQ) or EBSS (Additional file 2: Figure S3a and $\mathrm{b}$ ). Moreover, nutrient starvation failed to induce autophagy when ITPR1 was knocked down in cancer cells, as shown by confocal microscopy examination $48 \mathrm{~h}$ after mRFP-GFP-LC3 adenovirus vector transfection (Additional file 2: Figure S3c and d). Additionally, electron microscopy examination indicated that ITPR1 knockdown reduced the number of autophagic vesicles (Additional file 2: Figure S3e).

We next explored whether EGOT was involved in autophagy via ITPR1 in the presence of CQ or EBSS. As expected, overexpression of EGOT increased LC3-II/ LC3-I levels and reduced P62 expression in HeLa and breast cancer cells (Fig. 3a and b; Additional file 2: Figure S3f and g), while knockdown of EGOT reduced LC3-II/LC3-I levels and increased P62 expression (Fig. 3c and d). Meanwhile, overexpression of EGOT induced autophagy, as shown by the increases in mRFP-GFP-LC3 puncta accumulation and the number of autophagic vesicles (Fig. 3e and g; Additional file 2: Figure S3 h). In contrast, knockdown of EGOT caused the opposite changes (Fig. 3f and h). Moreover, EGOT-induced autophagy could be reversed by ITPR1 knockdown in HeLa and MCF7 cells (Fig. 3k; Additional file 2: Figure S3i). These results revealed that EGOT can at least partly convey the autophagic signal via ITPR1.

Furthermore, by analyzing CCLE data, we found that ITPR1 may impact paclitaxel sensitivity in tumor cells (Additional file 2: Figure S3j). The result was validated by experimental evidence showing that ITPR1 knockdown conferred protection to cancer cells from paclitaxel (Additional file 2: Figure S3k). Moreover, paclitaxel sensitivity induced by EGOT overexpression could be repressed by ITPR1 knockdown in vitro (Additional file 2: Figure S3 1). In addition, EGOT-overexpressing tumors exposed to paclitaxel treatment in vivo expressed high levels of ITPR1 and LC3B proteins (Fig. 3i; Additional file 2: Figure S3 $\mathrm{m}$ and $\mathrm{n}$ ). Consistently, breast cancer tissues with higher EGOT levels showed higher ITPR1 and LC3B levels in the HMUCC cohort, suggesting that the increased expression of EGOT may force a connection with the autophagic pathway via ITPR1 (Fig. 3j). These results indicate that EGOT enhances paclitaxel sensitivity through ITPR1 in human cancer.

\section{EGOT regulates ITPR 1 expression both in cis and in trans in human cancer}

Emerging evidence shows that lncRNAs can regulate gene expression and protein functions in trans or in cis [9]. Some lncRNAs are known to be involved in regulating protein synthesis or protein stability in mammalian cells [25]. We found that neither ITPR1 protein synthesis nor protein stability appeared to have any significant effect on the overexpression of EGOT via assays using the protein synthesis inhibitor cycloheximide (CHX) and the proteasome inhibitor MG132 (Additional file 2: Figure S4a and b).

By fractionated nuclear and cytoplasmic RNA analysis and RNA fluorescence in situ hybridization (RNA-FISH) examination in four cancer cell lines, we found that EGOT is mainly expressed in the nucleus (Fig. 4a and b; Additional file 2: Figure S4c and d), suggesting that $E G O T$ exerts its regulatory function for ITPR1 at the transcriptional level. Of note, ectopic expression of EGOT induced endogenous upregulation of pre-ITPR1 (Fig. 4c), while EGOT knockdown suppressed endogenous expression of pre-ITPR1 (Fig. 4d). Moreover, the stability of pre-ITPR1 mRNA was increased in EGOT-overexpressing cancer cells compared with that in control cells, as shown in the transcriptional inhibition experiments (Additional file 2: Figure S4e).

Given that EGOT is complementary to intronic sequences of the ITPR 1 and is transcribed in the antisense direction, we hypothesized that a double-stranded (ds) RNA forms between EGOT and pre-ITPR1. As expected, dual-RNA-FISH assay indicated that EGOT and pre-ITPR1 hybridized in the same nuclear foci (Fig. 4e). These foci were completely depleted upon treatment with RNase III, which degrades only dsRNA, but not with RNase A, which degrades only single-stranded (ss) RNA (Fig. 4e), thereby indicating the formation of dsRNA from the physical association of EGOT and pre-ITPR1 (Additional file 2: Figure S4f). Furthermore, through domain mapping, two exons of EGOT were divided into four fragments (Fig. 4f). Interestingly, EGOT fragment 2 (324-645 nucleotides) in exon 1 increases ITPR1 protein levels as well as ITPR1 and pre-ITPR 1 mRNA levels, whereas other fragments do not possess these capacities (Fig. $4 \mathrm{~g}$ and h). Taken together, these data indicate the pivotal roles of EGOT fragment 2 (324-645 nucleotides) in exon 1, which binds to pre-ITPR1 to control ITPR1 expression in cis in human cancer.

Accumulating evidence suggests that lncRNA activity is centered on its ability to bind and regulate epigenetic complexes of methylation, acetylation and phosphorylation $[26,27]$. Therefore, by using the Flag-MS2bp-MS2bs-based system (Additional file 2: Figure S4g) and, subsequently, mass spectrometry examination (Additional file 1: Table S5), hnRNPH1 was pulled down in this process, and the result was validated in four other cancer cell lines (Fig. 4i). RNA immunoprecipitation (RIP) further revealed that hnRNPH1 bound to both EGOT and pre-ITPR1 mRNA (Fig. 4j). Given the earlier evidence that hnRNPH1 interacts with RNA-binding proteins to improve alternative splicing [28], inhibition of hnRNPH1 expression may suppress alternative splicing of its target. As expected, knockdown of hnRNPH1 decreased ITPR1 
a

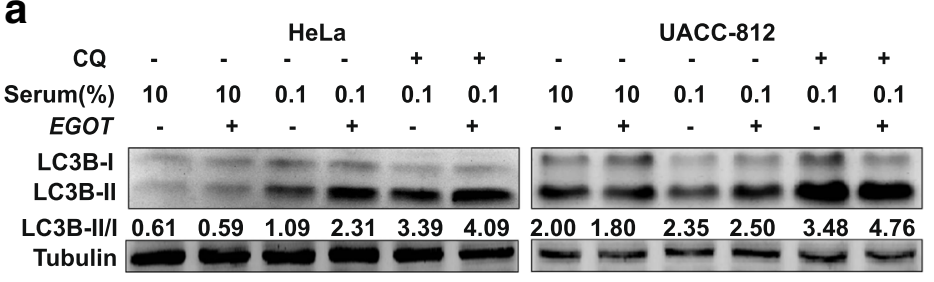
b b

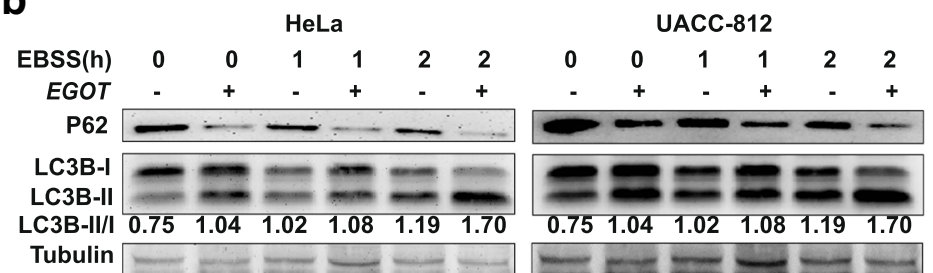

\section{C} CQ - $\quad \stackrel{\text { T47D }}{ }-\quad+\quad+$ $\begin{array}{lllllll}\text { Serum(\%) } & 10 & 10 & 0.1 & 0.1 & 0.1 & 0.1\end{array}$ EGOTKD - + $\quad$ - + + $\quad$ LC3B-I $=20$

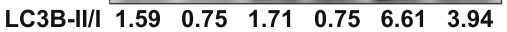
Tubulin $\ldots \ldots$ d

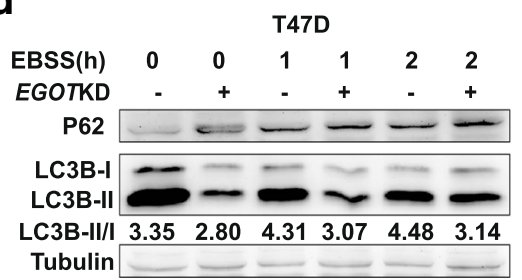

e
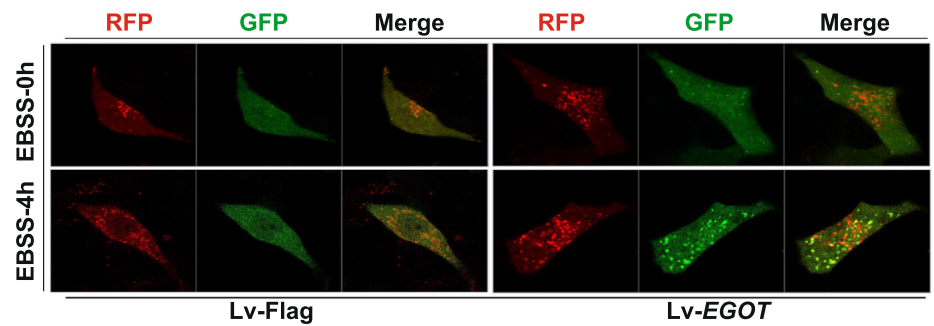

GFP Merge
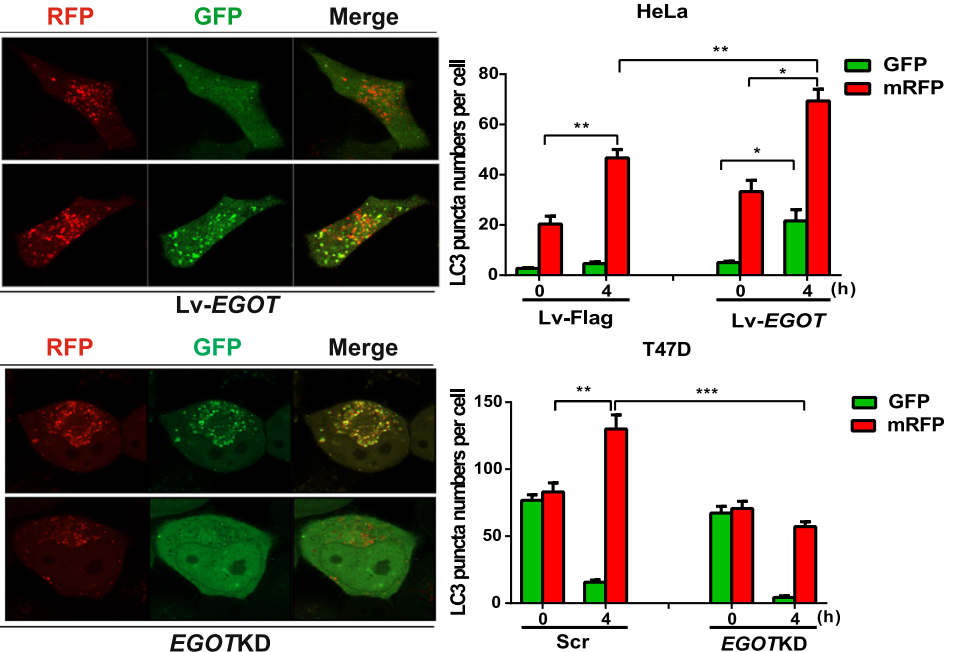

f

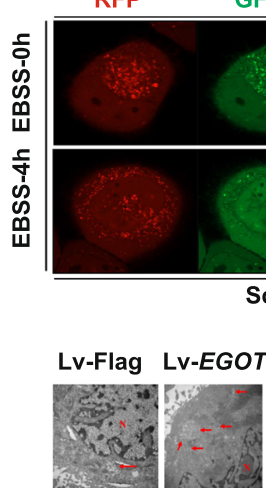

HeLa

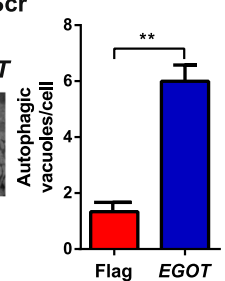

h

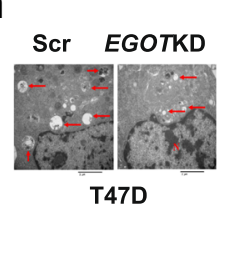

i

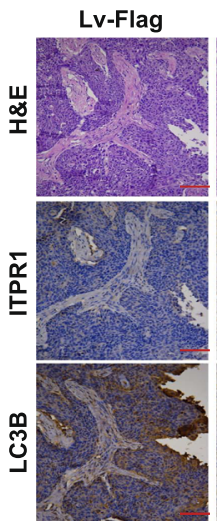

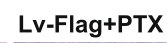

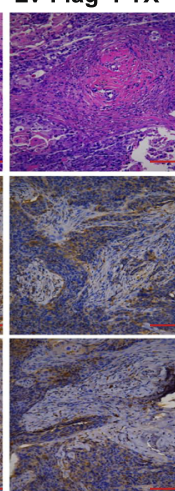

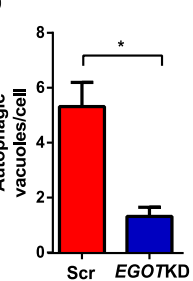

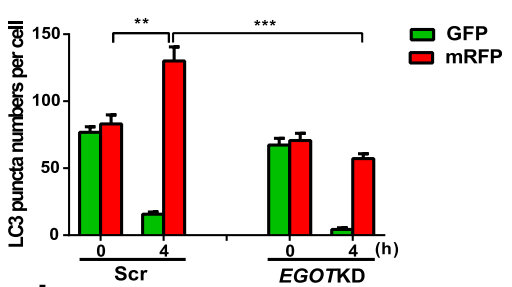
j
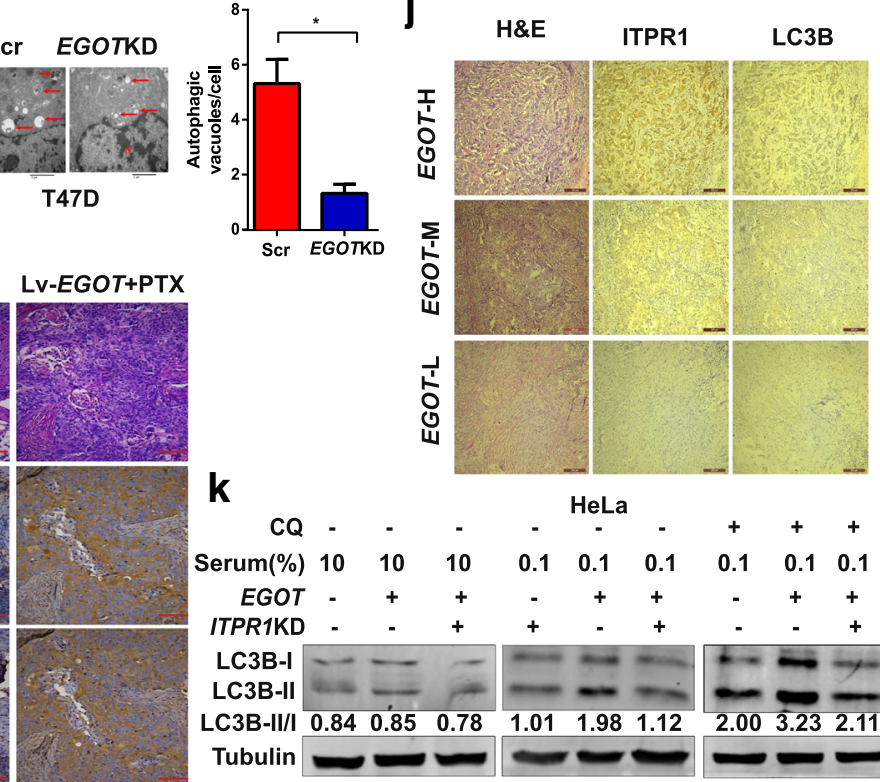

$\mathbf{K}$

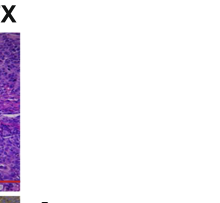
$\begin{array}{llllllllll}\text { Serum(\%) } & 10 & 10 & 10 & 0.1 & 0.1 & 0.1 & 0.1 & 0.1 & 0.1\end{array}$ $\begin{array}{rlllllllllllll}E G O T & - & + & + & - & + & + & - & + & + \\ \text { ITPR1KD } & - & + & + & - & + & - & - & +\end{array}$ LC3B-I - - - - - $\cdots$ LC3B-II $\div-\div-\infty-\infty-\infty$ \begin{tabular}{llllllllll}
\hline LC3B-II/I & 0.84 & 0.85 & 0.78 & 1.01 & 1.98 & 1.12 & 2.00 & 3.23 & 2.11 \\
\hline
\end{tabular} Tubulin $=$

Fig. 3 (See legend on next page.) 
(See figure on previous page.)

Fig. $3 E G O T$ induces autophagy to enhance paclitaxel sensitivity through ITPR1. a Western blot showing the effects of EGOT overexpression on LC3-II/LC3-I levels in HeLa (left) and UACC-812 cells (right) treated with CQ. b Western blot showing the effects of EGOT overexpression on LC3-II/ LC3-I levels and P62 expression in HeLa (left) and UACC-812 cells (right) treated with EBSS. c Western blot showing the effects of EGOT overexpression on LC3-II/LC3-I levels in T47D cells treated with CQ. d Western blot showing the effects of EGOT overexpression on LC3-II/LC3-I levels and P62 expression in HeLa (left) and UACC-812 cells (right) treated with EBSS. e, $\mathbf{f}$ Confocal microscopy showing the effects of EBSS incubation on mRFP-GFP-LC3 distribution in HeLa EGOT overexpression cells (e) or T47D EGOT knockdown cells (f) $48 \mathrm{~h}$ after mRFP-GFP-LC3 adenovirus transfection (10,000× magnification). $\mathbf{g}$, $\mathbf{h}$ Representative electron microscopy images and quantification of autophagic vacuoles in EGOT overexpression cells $(\mathbf{g})$ and EGOT knockdown cells $(\mathbf{h})$. Scale bar, $2 \mu \mathrm{m}$. Arrows depict autophagosomes, and the nucleus is denoted by N. $\mathbf{i}$ Representative hematoxylin and eosin (H\&E), ITPR1, and LC3B staining of orthotopic xenograft sections from UACC-812 EGOT overexpression and control groups treated with or without paclitaxel. Scale bar, $400 \mu \mathrm{m}$. $\mathbf{j}$ Representative H\&E, ITPR1 and LC3B staining in sections from 258 breast cancer tissues in HMUCC cohort. Scale bar, $200 \mu \mathrm{m}$. EGOT-H, EGOT-M, and EGOT-L represent high, medium, and low expression of EGOT, respectively. $\mathbf{k}$ Western blot showing LC3-II/LC3I levels in HeLa cells treated with CQ, different serum condition, EGOT overexpression or ITPR1 knockdown. Data are shown as the mean \pm s.d. Student's t-test was used for statistical analysis: ${ }^{*} P<0.05 ;{ }^{* *} P<0.01$; ${ }^{* *} P<0.001$; and ${ }^{* * * *} P<0.0001$. Data represent at least three independent experiments

mRNA and protein levels (Fig. $4 \mathrm{k}$ and $\mathrm{l}$ ). As EGOT fragment 2 (324-645 nucleotides) in exon 1 binds to pre-ITPR1 mRNA in cis (Fig. 4g), ectopic expression of EGOT fragment 2 (324-645) resulted in enrichment of hnRNPH1 by the RNA pull-down assays (Fig. $4 \mathrm{~m}$ ). hnRNPH1 has been reported to regulate alternative splicing by binding to specific motifs (GGGA) [29]. Moreover, mutation of the specific binding motifs (mut-A and mut-C) in EGOT fragment 2 led to the failure of hnRNPH1 enrichment (Fig. 4n). Altogether, these data demonstrated that hnRNPH1 is recruited by EGOT fragment 2 to enhance the alternative splicing of pre-ITPR 1 in trans.

\section{EGOT expression is transcriptionally regulated by stress in human cancer}

Through guilt-by-association analysis, we found that $E G O T$ is involved in stress-associated conditions (Additional file 1: Table S1). Stress, including hypoxia, estrogen depletion, radiotherapy, and chemotherapy, has been shown to affect autophagy induction [30-33]. Thus, we first aimed to determine whether EGOT is regulated by hypoxia. Hypoxia (1\% oxygen) treatment induced a sustained upregulation of $E G O T$ in a time-dependent manner in several cell types (Additional file 2: Figure S5a-e). Moreover, by analyzing previously published GEO datasets (GDS4121, GDS1453 and GDS723) involving different stress treatments, we found that EGOT was also upregulated in the absence of HGF stimulation or treatment with camptothecin or radiation (Additional file 2: Figure S5f-h). Furthermore, EGOT was upregulated in breast cancer patients who underwent neoadjuvant chemotherapy compared with its expression in patients who did not receive such therapy in the HMUCC cohort (Additional file 2: Figure S5i).

Estrogen (E2) is strongly related to breast cancer and has been shown to inhibit autophagy in a previous report [34]. Regarding the potential role of E2 in regulating EGOT expression, E2 indeed suppressed EGOT expression in a dose-dependent manner (Fig. 5a). Moreover, analysis from the GSE11324 dataset further certified the relationship of EGOT inhibition by E2, which was accordance with our results above (Additional file 2: Figure S6a). Concordantly, the expression levels of several positive control genes that are negatively regulated by E2, such as IRX4, GUSB, BCAS4 and MUC1, were decreased after E2 stimulation (Fig. 5b). In contrast, EGOT expression was induced in response to treatment with the anti-estrogen agents tamoxifen and fulvestrant (ICI) (Fig. 5c and Additional file 2: Figure S6b). In addition, knockdown of the estrogen receptor (ER) in MCF7 cells was shown to induce the expression of EGOT by $>10$-fold (Fig. 5d), while the expression of the positive control gene GREB1, which was reported to be positively regulated by E2, was decreased, and that of the IRX4, which was reported to be negatively regulated by E2, was increased (Additional file 2: Figure S6c). Furthermore, time course analysis demonstrated that E2 suppressed EGOT expression in a time-dependent manner (Fig. 5e). Moreover, dose-dependent inhibition of EGOT expression by E2 was also observed in other ER-positive T47D breast cancer cells (Additional file 2: Figure S6d). In all, these results demonstrate that $E G O T$ is transcriptionally inhibited by E2 in ER-positive breast cancer cells.

Next, we determined the molecular mechanism underlying such transcriptional repression. Estrogen-inducible NRIP1 acts as a nuclear receptor corepressor and interacts with AP-1 to directly repress target genes via ERE or AP-1 elements [35]. The expression of NRIP1 was induced, whereas EGOT expression was decreased after E2 treatment in MCF7 cells (Fig. 5f). As expected, transcriptional repression of EGOT by E2 was markedly reversed and the positive control gene GREB1 was upregulated in the presence of siNRIP1 (Fig. $5 \mathrm{~g}$ and h; Additional file 2: Figure S6e), demonstrating that NRIP1 is necessary and involved in this process. Furthermore, coimmunoprecipitation assays revealed that NRIP1 bound to AP-1 (Fig. 5i). Moreover, NRIP1 and AP-1 chromatin immunoprecipitation sequencing experiments showed that AP-1 and NRIP1 binding sites were enriched near the transcription 


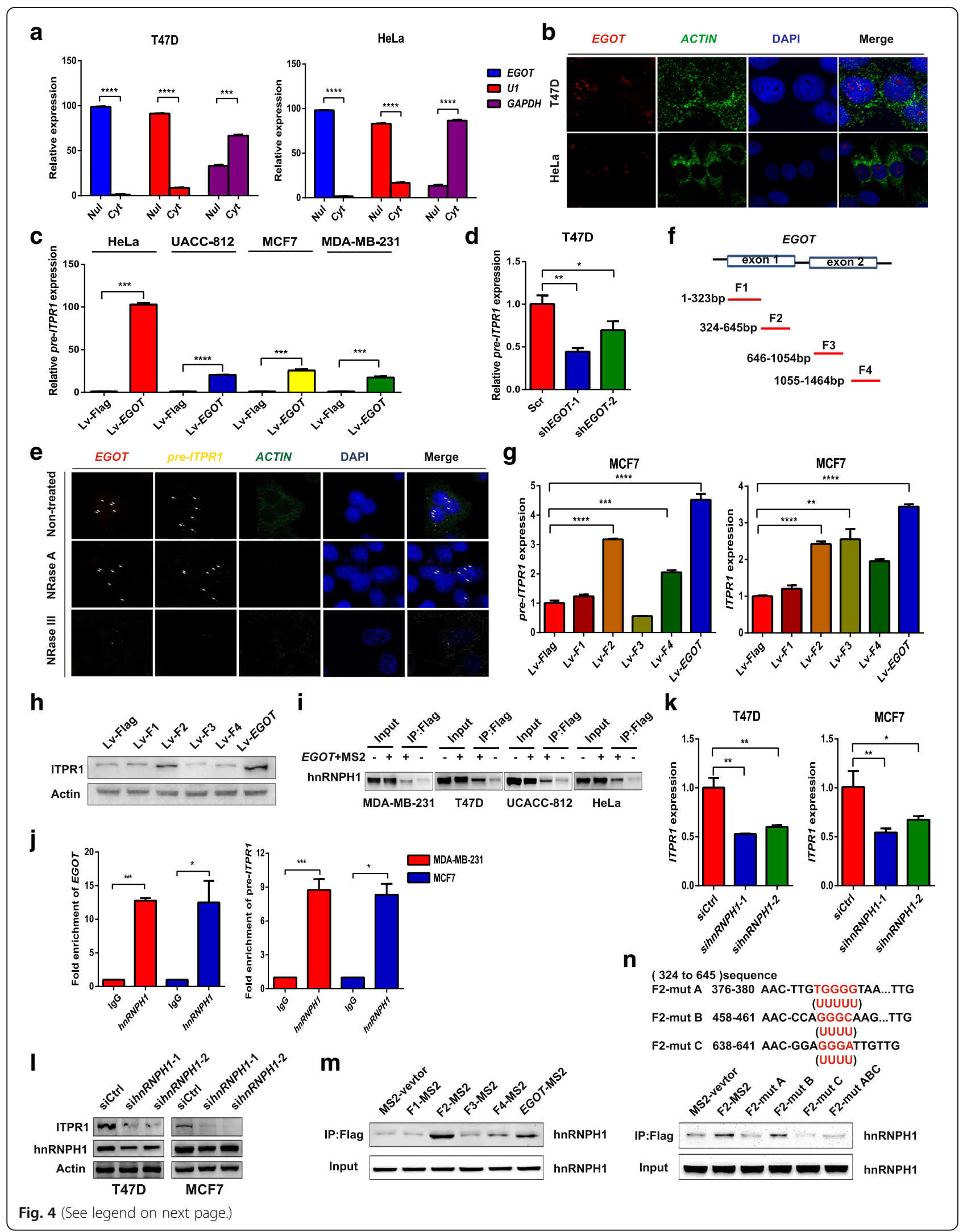


(See figure on previous page.)

Fig. 4 EGOT regulates ITPR1 expression in cis and in trans in human cancer. a Nuclear and cytoplasmic fractions of HeLa and T47D cells were subjected to qRT-PCR. U1 is the nuclear (Nul) positive control; GAPDH is the cytoplasmic (Cyt) positive control. b RNA-FISH performed in HeLa and T47D cells. EGOT probes are red; ACTIN probes are green; and ACTIN served as the positive control (1000 $\times$ magnification). c, $\mathbf{d}$ Expression of pre-ITPR1 in EGOT overexpression and knockdown cells by qRT-PCR. e Combined RNase resistance and dual-RNA-FISH analysis. Before hybridization, T47D cells were treated with RNase A or RNase III. Hybridization was performed with specific probes against EGOT and ITPR1 transcripts. Nuclei are stained with DAPI. EGOT probes are red; ITPR1 probes are yellow; and ACTIN is the positive control. Arrows indicate foci (1000 $\times$ magnification). f Domain mapping of the EGOT transcript. $\mathbf{g}$, $\mathbf{h}$ Expression of pre-ITPR1 and ITPR1 mRNA (g) and protein (h) in MCF7 cells transfected with different fragments of EGOT lentivirus. i An RNA pull-down assay was conducted using the Flag-MS2bp-MS2bs-based system followed by western blotting of lysates from MDA-MB-231, T47D, and UACC-812 cells after transfection with MS2-EGOT and MS2-vector (control). j Antibodies against hnRNPH1 were used for RIP, followed by qRT-PCR, in MCF7 and MDA-MB-231 cells. $\mathbf{k}$, I ITPR1 mRNA expression was analyzed by qRT-PCR after hnRNPH1 knockdown via siRNAs (k), followed by western blotting (I). $\mathbf{m}$ The RNA pull-down assay was conducted using the Flag-MS2bp-MS2bs-based system in T47D cells after transfection with MS2-EGOT lentivirus along with different fragments of EGOT lentivirus, followed by western blotting. $\mathbf{n}$ Western blot of hnRNPH1 pulled down by F2-MS2 and mutated F2 RNA in 293 T cells. The red underlined sequences indicate the potential binding sites that were mutated into Us in F2, while the $\mathrm{A}, \mathrm{B}$ and $\mathrm{C}$ binding sites were mutated at the same time to F2-mut ABC. Data are shown as the mean \pm s.d. Student's t-test was used for statistical analysis: ${ }^{*} P<0.05 ;{ }^{*} P<0.01$; ${ }^{* *} P<0.001$; and **** $P<0.0001$. Data represent at least three independent experiments

start sites $(-5 \mathrm{~kb}$ to $5 \mathrm{~kb})$ of target genes (Additional file 2: Figure S6f). Interestingly, significant enrichment peaks for AP-1 and NRIP1 were detected at a genomic locus (chr 3: 4757428-4,757,590) upstream of the transcription start site of EGOT (Fig. 5j). Next, the binding of AP-1 and NRIP1 to the promoter region of EGOT was validated by ChIP-quantitative PCR (ChIP-PCR) (Fig. 5k). Taken together, our results indicated that NRIP1 physically interacts with AP-1 and transcriptionally represses the expression of EGOT in presence of E2 (Fig. 5l).

\section{EGOT/ITPR1 expression is associated with a favorable prognosis and enhances paclitaxel sensitivity in human cancer}

The prognostic role of EGOT and ITPR1 was finally examined in the HMUCC and public cohort data. High expression of EGOT was associated with favorable OS and RFS) in breast cancer patients, while loss of ITPR1 indicated worse RFS in the HMUCC cohort (Fig. 6a and b; Additional file 2: Figure S7a). These results were then validated in 14,586 cancer patients in TCGA and ICGC human cancer cohorts, and patients with high expression levels of EGOT showed favorable OS and RFS in both of 33 human cancer types and breast cancer cohorts (Fig. 6c and d; Additional file 2: Figure S7b and c), while patients with high ITPR1 expression showed favorable OS in the human cancer cohort (Additional file 2: Figure S7d and e).

Regarding paclitaxel sensitivity, breast cancer patients who were treated with paclitaxel and exhibited high expression levels of EGOT demonstrated better OS and RFS than patients with low expression levels of EGOT in the HMUCC cohort (Fig. 6e and f). Moreover, the results were further validated in TCGA breast cancer patients treated with paclitaxel, which showed that high expression of EGOT was also associated with favorable OS, in accordance with our study (Fig. 6g). Similarly, loss of ITPR1 was associated with poor RFS in breast cancer patients treated with paclitaxel in the HMUCC cohort (Additional file 2: Figure S7f). More importantly, breast cancer patients with high expression of EGOT achieved a higher complete pathological response ratio than patients with low expression of EGOT when treated with paclitaxel-containing adjuvant chemotherapy regimens in the HMUCC cohort (Fig. 6h). Therefore, EGOT/ITPR1 expression is associated with a favorable prognosis and enhances paclitaxel sensitivity in human cancer.

\section{Discussion}

Regarding the function of Ai-lncRNA EGOT, we first reported that low levels of EGOT expression were significantly correlated with increased tumor size, lymph node metastasis, and Ki-67 expression in human breast cancer [14]. In this study, we identified an uncharacterized lncRNA, EGOT, and its roles in enhancing paclitaxel sensitivity by triggering autophagosome accumulation. Mechanistically, EGOT regulates ITPR1 expression in cis and in trans, leading to the activation of autophagy signaling. We also found that EGOT is transcriptionally regulated by various stress conditions that are associated with paclitaxel resistance. Overall, we provide compelling evidence that in response to stress, EGOT activates autophagy via ITPR1, which sensitizes paclitaxel cytotoxicity in human cancer (Additional file 2: Figure S8).

Several studies have investigated the role of macrophages in decreasing the cytotoxic effects of chemotherapy and demonstrated a therapeutic window for autophagy inhibition in cancer therapy and prevention [36, 37]. Paradoxically, a previous study showed that autophagy enhances paclitaxel-induced cell death and that reduced autophagy may contribute to clinical chemotherapeutic resistance in primary breast tumors [6]. Of note, paclitaxel blocks the activation of PI3K and Vps34 and inhibits the movement and maturation of autophagosomes to induce cell death [6]. In vitro, autophagosome accumulation sensitizes cells to 


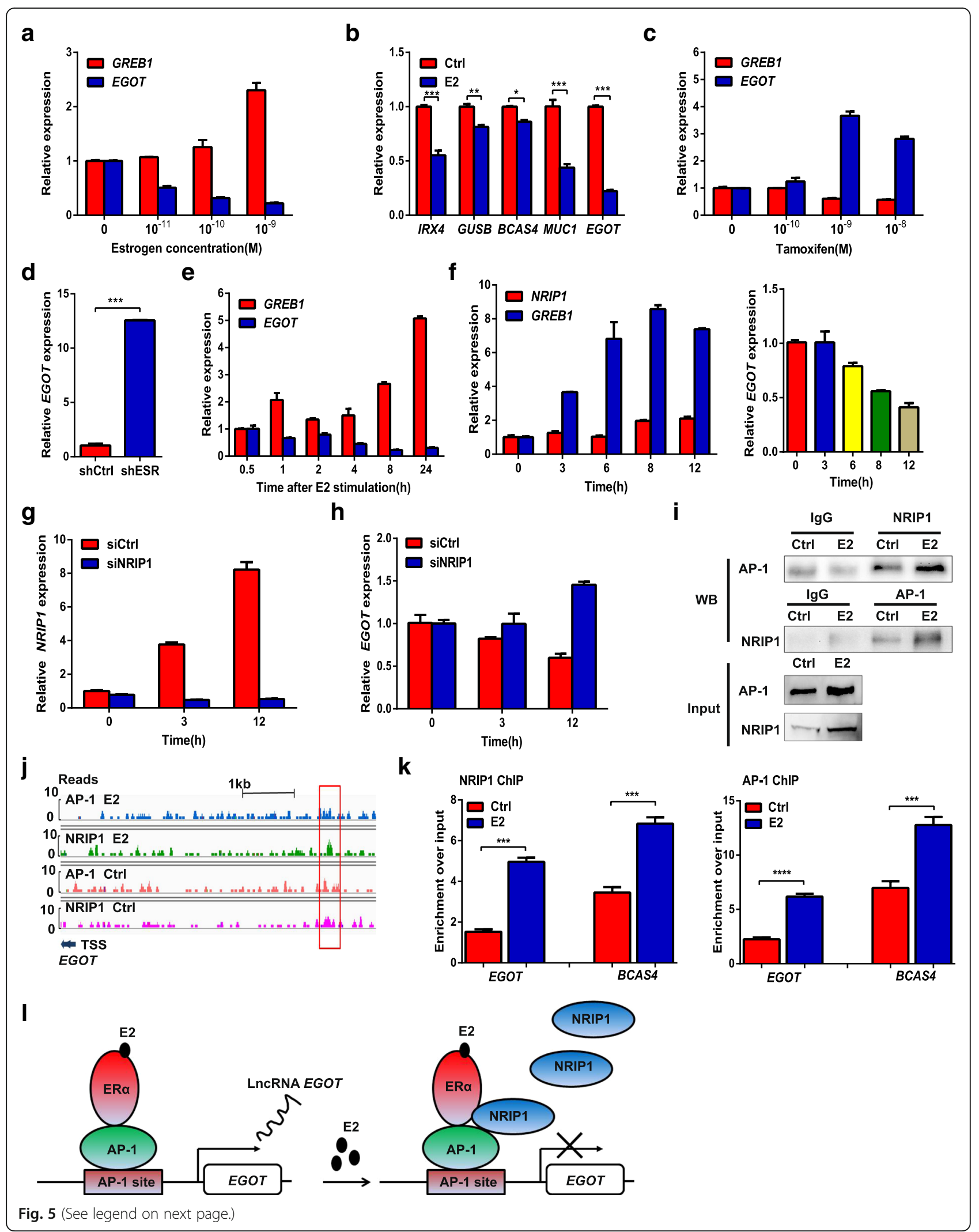




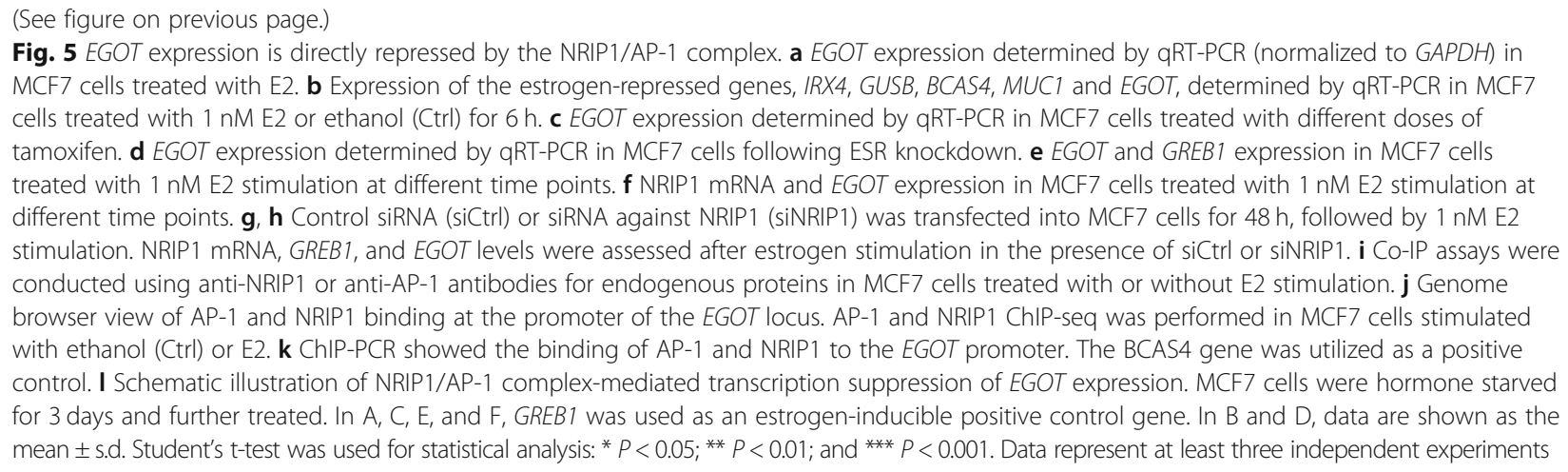

paclitaxel toxicity [6]. Using in vivo and in vitro experiments in this study, we found that EGOT overexpression activated autophagy signaling, which sensitizes cells to paclitaxel, whereas EGOT knockdown has the opposite effect, in accordance with this previous study. Mechanistically, EGOT upregulates the expression of ITPR1, which promotes autophagosome accumulation and sensitizes cells to paclitaxel toxicity, thereby highlighting a novel function for these molecules.

Loss or low expression of autophagy-related genes, indicating a decrease of autophagic flux, was associated with poor prognosis $[38,39]$. High expression of EGOT is significantly associated with favorable OS and RFS in patients in the HMUCC cohort and TCGA and ICGC public datasets. Furthermore, recent study showed that autophagy-related genes/proteins could be possible predictive markers for paclitaxel efficacy in the clinic [6]. High expression of $E G O T$ is significantly associated with enhanced paclitaxel sensitivity in patients in the HMUCC cohort and TCGA and ICGC public datasets, while the lack of EGOT expression indicates resistance to paclitaxel therapy. In addition, high expression of EGOT indicated an increased complete pathological response ratio following treatment with paclitaxel-containing adjuvant chemotherapy regimens, in contrast to patients with low EGOT expression in the HMUCC cohort. However, paclitaxel-containing adjuvant chemotherapy sensitivity has only been evaluated in a small study cohort; thus, a multicenter clinical trial with a larger patient number will be performed in a future study to validate these results. Hence, our study provides a proof of concept showing that EGOT may be a predictive marker of the clinical efficacy of paclitaxel treatment.

LncRNAs can function in cis to regulate the expression of neighboring genes or in trans to carry out many roles by various modes [9]. Compared to other category of long non-coding RNAs, the function of Ai-lncRNAs including EGOT is still largely unknown. In this study, we clearly demonstrated that EGOT regulates ITPR1 expression both in cis and in trans. On one hand, EGOT regulates ITPR1 levels via a unique regulatory mechanism involving the formation of a pre-ITPR1/EGOT dsRNA that induces pre-ITPR1 accumulation to increase ITPR1 protein expression in cis. However, we should mention that although it was initially proposed that lncRNA mainly functions to regulate neighboring gene transcription, other studies have shown that many lncRNAs do not exert such function [40, 41]. Thus, whether EGOT regulates any other neighboring gene transcription in cis awaits further investigation. Furthermore, EGOT recruits hnRNPH1 to promote ITPR1 expression in trans. hnRNPH1, a splicing factor, has been shown to regulate alternative splicing and polyadenylation [42, 43]. EGOT fragment 2 (324-645 nucleotides) in exon 1 was found to bind pre-ITPR1 mRNA and the hnRNPH1 protein, thereby mediating alternative splicing of pre-ITPR1 mRNA. hnRNPH1 has been shown to bind G-rich sequences interspersed with adenosines around exons $[28,42]$. ITPR 1 contains 59 exons and multiple GGGA/C/G motifs distributed in these exons. Thus, we propose that hnRNPH1 mediates pre-ITPR1 splicing in human cancer by binding these GGGA/C/G motifs. Taken together, these findings broaden the understanding of the autophagy-associated lncRNA landscape and provide novel insights into the exploration of the mechanism of autophagy regulation in human cancer.

Our data also show that $E G O T$ is positively and negatively transcriptionally regulated by hypoxia and estrogen-related stress, respectively, in human cancer. Studies have shown that hypoxia can promote tumor invasion, metastasis, autophagy, and angiogenesis, can regulate tumor metabolism and is closely related to the poor prognosis of cancers $[44,45]$. Hypoxia is associated with the expression of numerous noncoding RNAs, including miRNAs and lncRNAs [46]. In this study, EGOT was induced in vitro by subjecting different cancer cell lines to hypoxia. Apart from its positive regulation by hypoxia, EGOT can be transcriptionally repressed by estrogen. Estrogen, a paracrine mediator throughout life, is an important factor in tumorigenesis in humans. Estrogen binds to estrogen receptors to control vast gene networks that are involved in glycolysis, glutaminolysis, oxidative 


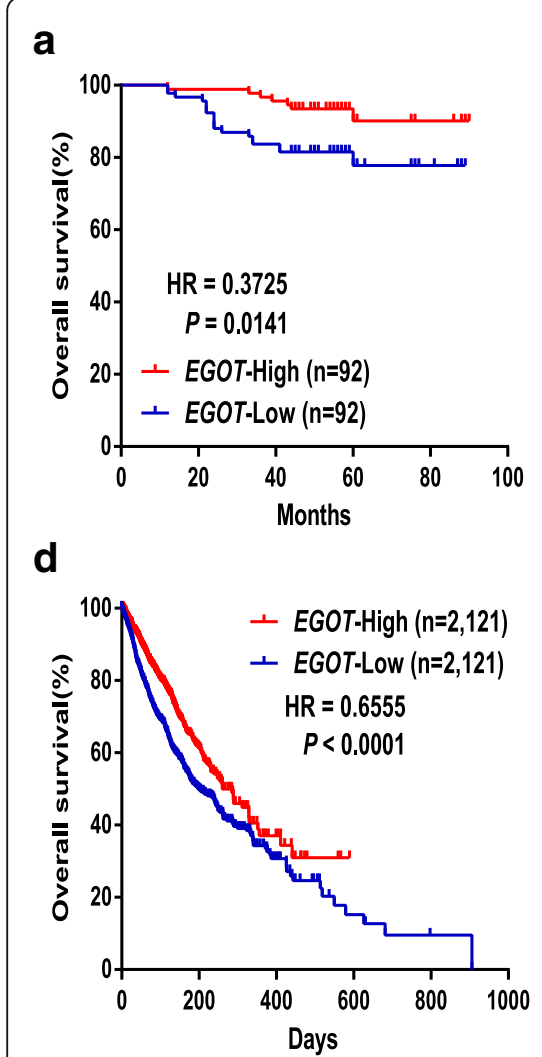

b

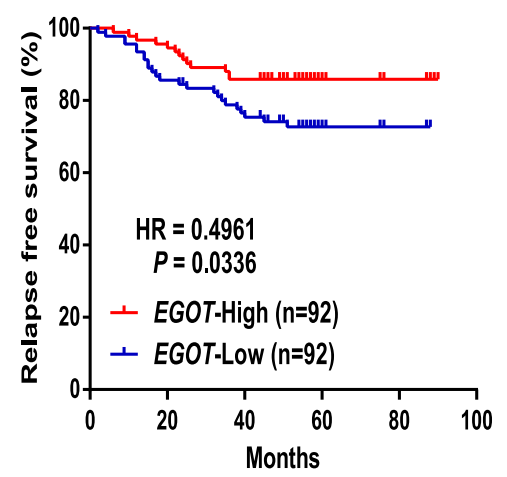

e

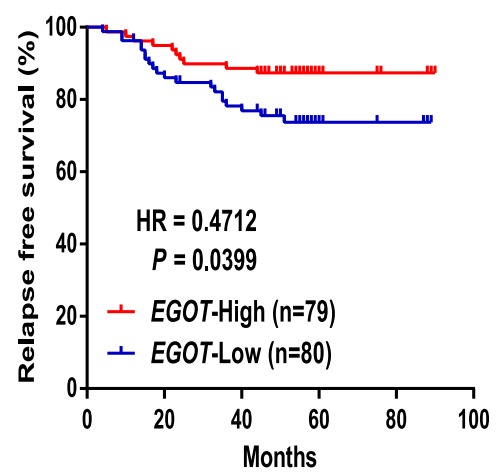

C

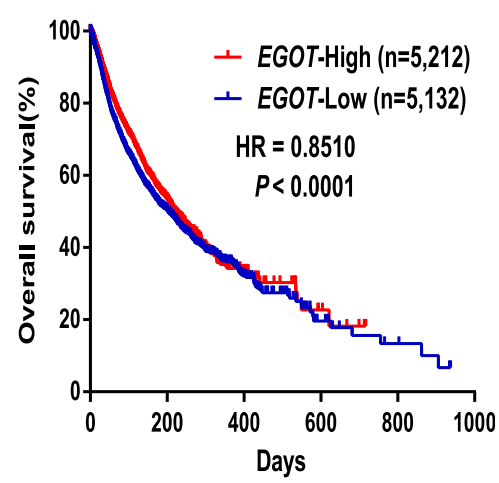

f

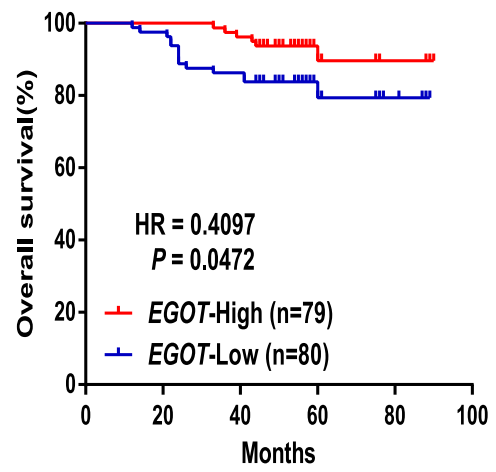

g

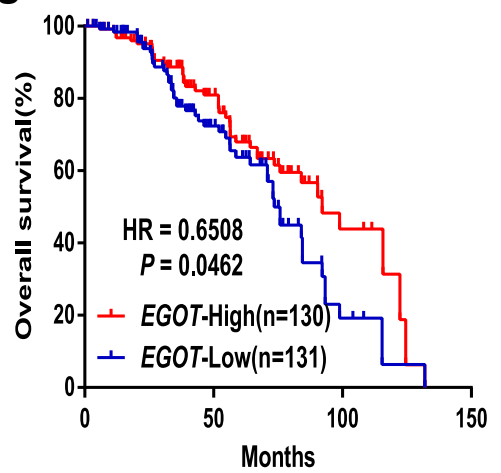

h

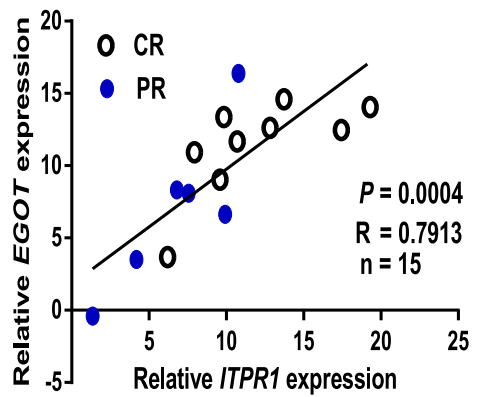

\begin{tabular}{lccc}
\hline EGOT & High & Low & $P$ \\
\hline CR & 7 & 1 & 0.0406 \\
PR & 2 & 5 & \\
\hline
\end{tabular}

Fig. 6 EGOT/ITPR1 expression is associated with a favorable prognosis and enhances paclitaxel sensitivity in human cancer. a, b Kaplan-Meier analyses of the relationships between EGOT expression and OS (a) or RFS (b) in breast cancer patients in the HMUCC cohort. $\mathbf{c}$, $\mathbf{d}$ Kaplan-Meier analyses of the relationships between EGOT expression and OS among 33 cancer types in TCGA (c) and ICGC cohorts (d). e, f Kaplan-Meier analyses of the relationships between EGOT expression and OS (e) or RFS (f) in breast cancer patients treated with paclitaxel in the HMUCC cohort. $\mathbf{g}$ Kaplan-Meier analyses of the relationship between EGOT expression and OS in breast cancer patients treated with paclitaxel in the TCGA cohort. $\mathbf{h}$ The correlations between EGOT expression and ITPR1 mRNA expression were measured in 15 breast cancer patients treated with paclitaxel-containing adjuvant chemotherapy regimens (left). EGOT and ITPR1 levels (normalized to GAPDH) were subjected to Pearson's correlation analysis. Correlations between EGOT expression and a pathological response were determined by the chi-square test (right). A complete pathological response is denoted by $C R$, while a partial response is denoted by PR. The median expression level was used as the cut-off value. Patients with EGOT expression values below the 50th percentile were classified as EGOT-Low. Patients with EGOT expression values above the 50th percentile were classified as EGOT-High

phosphorylation, nutrient sensing and biosynthesis pathways in cancer [47]. Several lncRNAs have been shown to be regulated by estrogen in breast cancer [22, 48]. In this study, EGOT was found to be a direct target of AP-1/ NRIP1-mediated transcriptional repression complex in the presence of estrogen. Previous studies have shown that NRIP1 is an obligate co-factor of the estrogen receptor, and germline single-nucleotide polymorphisms (SNPs) near NRIP1 have been associated with ER-positive breast cancer [49]. Estrogen mediates NRIP1 induction, 
which subsequently interacts with estrogen receptor AP-1 complexes to directly repress adjacent target genes, including BCAS4, IRX4, GUSB and MUC1 [35]. In this study, EGOT was inhibited by estrogen at physiological concentrations $\left(0-10^{-9} \mathrm{M}\right)$, which led to a gradual decrease in EGOT expression; meanwhile NRIP1 expression was gradually increased with time. Knockdown of NRIP1 reversed the estrogen-mediated transcriptional repression of EGOT expression. Interestingly, a previous study reported that the expression of ITPR1 protein decreased in an estrogen receptordependent manner and that the growth of MCF7 cells induced by estrogen was sensitive to pharmacological inhibitors of ITPR1 [16]. Our study provides a proof of concept indicating that $E G O T$ is transcriptionally repressed by the AP-1/NRIP1 complex in the presence of estrogen and that this axis may send regulatory feedback signals to repress the excessive activation of autophagy.

In conclusion, these findings broaden comprehensive understanding of the biology function of Ai-lncRNAs. Moreover, our findings demonstrate that EGOT my act as a clinical biomarker of paclitaxel response and that proper regulation of EGOT may be a novel synergistic strategy for enhancing paclitaxel sensitivity, thereby enhancing its clinical benefits for cancer patients.

\section{Additional files}

Additional file 1: Table S1. Lists of primers. Lists including PCR primers used in this study, primers used for shRNAs and siRNA sequences. Table S2. Details of the gene sequence probe sets. Table S3. Guilt-by-association analysis in breast cancer data from TCGA. Table S4. Pan-cancer data of 33 cancer contexts in TCGA. All cancer IDs and patient numbers are listed. Table S5. Protein mass spectrometry analysis in MDA-MB-231. RNA pull-down assays using the Flag-MS2bp-MS2bs-based system (Additional file 2: Figure S4F), followed by mass spectrometry in MDA-MB-231 cells. Raw data listing all identified proteins and all peptides from each sample. (ZIP $9889 \mathrm{~kb}$ )

Additional file 2: Figure S1. EGOT enhances paclitaxel sensitivity in human cancer. Figure S2. EGOT is positively related to ITPR1 and induces ITPR1 expression. Figure S3. EGOT induces autophagy to enhance paclitaxel sensitivity through ITPR1. Figure S4. EGOT regulates ITPR1 expression in cis and in trans in human cancer. Figure $\mathbf{S 5}$. EGOT is transcriptionally regulated by stress stimuli in human cancer. Figure S6. EGOT is directly repressed by the NRIP1/AP-1 complex. Figure S7. EGOT/ ITPR1 is associated with a favorable prognosis and enhances paclitaxel sensitivity in human cancer. Figure $\mathbf{5 8}$. Schematic illustration of the possible mechanisms involved in this study. (ZIP $9814 \mathrm{~kb}$ )

\section{Acknowledgements}

The authors thank the patients, study investigators, and staff who participated in this study.

\section{Funding}

This work was supported by funding from a specific research fund for the public service sector, National Health and Family Planning Commission of the People's Republic of China (Grant Number 201402003), the National Key Technology Support Program (Grant Number 2014BAl09B08), the Project Nn10 of Harbin Medical University Cancer Hospital (Grant Number Nn102017-02), the National Natural Science Foundation of China (Grant Number 81602323, 81872149), the Wu Lien-teh Science Foundation of Harbin Medical University (Grant Number WLD-QN1706), the Distinguished Young Scholars of Harbin Medical University Cancer Hospital (Grant Number JCQN2018-03) and the Young Elite Training Foundation Grant of Harbin Medical University Cancer Hospital (Grant Number JY2016-02), the Postdoctoral Scientific Research Developmental Fund of Heilongjiang (Grant Number LBH-Q18079), the National Natural Science Foundation of China (Grant Number 81802649) and Haiyan Grant of Harbin Medical University Cancer Hospital (Grant Number JJQN2018-05).

\section{Availability of data and materials}

Raw sequencing and processed RNASeq data from this study have been deposited into the NCBI GEO database under accession number GSE71651 (http://www.ncbi.nlm.nih.gov/geo/query/acc.cgi?token = obcxosaur xoppwx $\&$ acc $=$ GSE71651). ChIP-seq data were deposited in the NCBI SRA: SRP149488 (https://www.ncbi.nlm.nih.gov/sra/SRP149488).

\section{Authors' contributions}

D. P. and S.P.X. designed the study and performed the bioinformatics analyses. P.Y.W., J. Z., S.Y.S., J.F.Z. and H.W. performed the experiments. Q.W. W.W.Y., K.Q. and H.B.X. performed in vivo experiments. D. P. and S.P.X. supervised the study and wrote the manuscript. All authors have read and approved the manuscript.

\section{Ethics approval and consent to participate}

All patients consented to an institutional review board-approved protocol that allows comprehensive analysis of tumor samples (Ethics committee of Harbin Medical University). This study conforms to the Declaration of Helsinki.

\section{Consent for publication}

Written informed consent for publication was obtained from the patients. All authors have agreed to publish this manuscript.

\section{Competing interests}

The authors declare that they have no competing interests.

\section{Publisher's Note}

Springer Nature remains neutral with regard to jurisdictional claims in published maps and institutional affiliations.

\section{Author details}

'Department of Breast Surgery, Harbin Medical University Cancer Hospital, 150 Haping Road, Harbin 150081, China. ${ }^{2}$ Department of Pathology, Harbin Medical University, Harbin, China. ${ }^{3}$ Heilongjiang Academy of Medical Sciences, 157 Baojian Road, Harbin 150086, China.

Received: 13 March 2019 Accepted: 8 April 2019 Published online: 18 April 2019

\section{References}

1. Yu Y, Gaillard S, Phillip JM, Huang TC, Pinto SM, Tessarollo NG, Zhang Z, Pandey A, Wirtz D, Ayhan A, et al. Inhibition of spleen tyrosine kinase potentiates paclitaxel-induced cytotoxicity in ovarian Cancer cells by stabilizing microtubules. Cancer Cell. 2015;28:82-96.

2. Luo Y, Li D, Ran J, Yan B, Chen J, Dong X, Liu Z, Liu R, Zhou J, Liu M. Endbinding protein 1 stimulates paclitaxel sensitivity in breast cancer by promoting its actions toward microtubule assembly and stability. Protein Cell. 2014;5:469-79.

3. Mizushima N, Komatsu M. Autophagy: renovation of cells and tissues. Cell. 2011;147:728-41. 
4. Jeon YJ, Khelifa S, Ratnikov B, Scott DA, Feng Y, Parisi F, Ruller C, Lau E, Kim $H$, Brill LM, et al. Regulation of glutamine carrier proteins by RNF5 determines breast cancer response to ER stress-inducing chemotherapies. Cancer Cell. 2015;27:354-69.

5. Domenech E, Maestre C, Esteban-Martinez L, Partida D, Pascual R, Fernandez-Miranda G, Seco E, Campos-Olivas R, Perez M, Megias D, et al. AMPK and PFKFB3 mediate glycolysis and survival in response to mitophagy during mitotic arrest. Nat Cell Biol. 2015;17:1304-16.

6. Veldhoen RA, Banman SL, Hemmerling DR, Odsen R, Simmen T, Simmonds AJ, Underhill DA, Goping IS. The chemotherapeutic agent paclitaxel inhibits autophagy through two distinct mechanisms that regulate apoptosis. Oncogene. 2013;32:736-46.

7. Cech TR, Steitz JA. The noncoding RNA revolution-trashing old rules to forge new ones. Cell. 2014;157:77-94.

8. Batista PJ, Chang HY. Long noncoding RNAs: cellular address codes in development and disease. Cell. 2013;152:1298-307.

9. Joung J, Engreitz JM, Konermann S, Abudayyeh OO, Verdine VK, Aguet F, Gootenberg JS, Sanjana NE, Wright JB, Fulco CP, et al. Genome-scale activation screen identifies a IncRNA locus regulating a gene neighbourhood. Nature. 2017;548:343-6.

10. Zhang J, Wang P, Wan L, Xu S, Pang D. The emergence of noncoding RNAs as Heracles in autophagy. Autophagy. 2017;13:1004-24.

11. Lin A, Hu Q, Li C, Xing Z, Ma G, Wang C, Li J, Ye Y, Yao J, Liang K, et al. The LINK-A IncRNA interacts with Ptdlns(3,4,5)P3 to hyperactivate AKT and confer resistance to AKT inhibitors. Nat Cell Biol. 2017;19:238-51.

12. Echevarria W, Leite MF, Guerra MT, Zipfel WR, Nathanson MH. Regulation of calcium signals in the nucleus by a nucleoplasmic reticulum. Nat Cell Biol. 2003:5:440-6

13. Adkins CE, Morris SA, De Smedt H, Sienaert I, Torok K, Taylor CW. Ca2+ -calmodulin inhibits Ca2+ release mediated by type-1, -2 and -3 inositol trisphosphate receptors. Biochem J. 2000;345(Pt 2):357-63.

14. Xu SP, Zhang JF, Sui SY, Bai NX, Gao S, Zhang GW, Shi QY, You ZL, Zhan C, Pang D. Downregulation of the long noncoding RNA EGOT correlates with malignant status and poor prognosis in breast cancer. Tumour Biol. 2015;36: 9807-12.

15. Hall DP, Cost NG, Hegde S, Kellner E, Mikhaylova O, Stratton Y, Ehmer B, Abplanalp WA, Pandey R, Biesiada J, et al. TRPM3 and miR-204 establish a regulatory circuit that controls oncogenic autophagy in clear cell renal cell carcinoma. Cancer Cell. 2014;26:738-53.

16. Fu NY, Sukumaran SK, Yu VC. Inhibition of ubiquitin-mediated degradation of MOAP-1 by apoptotic stimuli promotes Bax function in mitochondria. Proc Natl Acad Sci U S A. 2007;104:10051-6.

17. Akerman I, Tu Z, Beucher A, Rolando DM, Sauty-Colace C, Benazra M, Nakic N, Yang J, Wang H, Pasquali L, et al. Human pancreatic beta cell IncRNAs control cell-specific regulatory networks. Cell Metab. 2017;25:400-11.

18. Shan L, Zhou X, Liu X, Wang Y, Su D, Hou Y, Yu N, Yang C, Liu B, Gao J, et al. FOXK2 elicits massive transcription repression and suppresses the hypoxic response and breast Cancer carcinogenesis. Cancer Cell. 2016;30:708-22.

19. Wang K, Liu CY, Zhou LY, Wang JX, Wang M, Zhao B, Zhao WK, XU SJ, Fan $\mathrm{LH}$, Zhang XJ, et al. APF IncRNA regulates autophagy and myocardial infarction by targeting miR-188-3p. Nat Commun. 2015;6:6779.

20. Natale F, Rapp A, Yu W, Maiser A, Harz H, Scholl A, Grulich S, Anton T, Horl $D$, Chen $W$, et al. Identification of the elementary structural units of the DNA damage response. Nat Commun. 2017:8:15760.

21. Xu TP, Wang YF, Xiong WL, Ma P, Wang WY, Chen WM, Huang MD, Xia R, Wang R, Zhang EB, et al. E2F1 induces TINCR transcriptional activity and accelerates gastric cancer progression via activation of TINCR/STAU1/ CDKN2B signaling axis. Cell Death Dis. 2017;8:e2837.

22. Xue X, Yang YA, Zhang A, Fong KW, Kim J, Song B, Li S, Zhao JC, Yu J. LnCRNA HOTAIR enhances ER signaling and confers tamoxifen resistance in breast cancer. Oncogene. 2016:35:2746-55.

23. Xu S, Kong D, Chen Q, Ping Y, Pang D. Oncogenic long noncoding RNA landscape in breast cancer. Mol Cancer. 2017;16:129.

24. Salameh A, Lee AK, Cardo-Vila M, Nunes DN, Efstathiou E, Staquicini FI, Dobroff AS, Marchio S, Navone NM, Hosoya H, et al. PRUNE2 is a human prostate cancer suppressor regulated by the intronic long noncoding RNA PCA3. Proc Natl Acad Sci U S A. 2015;112:8403-8.

25. Huang S, Lu W, Ge D, Meng N, Li Y, Su L, Zhang S, Zhang Y, Zhao B, Miao J. A new microRNA signal pathway regulated by long noncoding RNA TGFB2OT1 in autophagy and inflammation of vascular endothelial cells. Autophagy. 2015;11:2172-83.
26. Amicone L, Citarella F, Cicchini C. Epigenetic regulation in hepatocellular carcinoma requires long noncoding RNAs. Biomed Res Int. 2015;2015:473942.

27. Mercer TR, Mattick JS. Structure and function of long noncoding RNAs in epigenetic regulation. Nat Struct Mol Biol. 2013;20:300-7.

28. Li X, Qian X, Peng LX, Jiang Y, Hawke DH, Zheng Y, Xia Y, Lee JH, Cote G, Wang $\mathrm{H}$, et al. A splicing switch from ketohexokinase- $\mathrm{C}$ to ketohexokinase-a drives hepatocellular carcinoma formation. Nat Cell Biol. 2016;18:561-71.

29. Chen M, David CJ, Manley J. Concentration-dependent control of pyruvate kinase M mutually exclusive splicing by hnRNP proteins. Nat Struct Mol Biol. 2012;19:346-54.

30. Amaravadi RK, Yu D, Lum JJ, Bui T, Christophorou MA, Evan Gl, ThomasTikhonenko A, Thompson CB. Autophagy inhibition enhances therapyinduced apoptosis in a Myc-induced model of lymphoma. J Clin Invest. 2007;117:326-36.

31. Sergin I, Evans TD, Bhattacharya S, Razani B. Hypoxia in plaque macrophages: a new danger signal for interleukin-1beta activation? Circ Res. 2014;115:817-20.

32. Vijayaraghavan S, Karakas C, Doostan I, Chen X, Bui T, Yi M, Raghavendra AS, Zhao Y, Bashour SI, Ibrahim NK, et al. CDK4/6 and autophagy inhibitors synergistically induce senescence in Rb positive cytoplasmic cyclin $\mathrm{E}$ negative cancers. Nat Commun. 2017:8:15916.

33. Bilanges B, Alliouachene S, Pearce W, Morelli D, Szabadkai G, Chung YL, Chicanne G, Valet C, Hill JM, Voshol PJ, et al. Vps34 PI 3-kinase inactivation enhances insulin sensitivity through reprogramming of mitochondrial metabolism. Nat Commun. 2017;8:1804.

34. Tyson JJ, Baumann WT, Chen C, Verdugo A, Tavassoly I, Wang Y, Weiner LM, Clarke R. Dynamic modelling of oestrogen signalling and cell fate in breast cancer cells. Nat Rev Cancer. 2011;11:523-32.

35. Carroll JS, Meyer CA, Song J, Li W, Geistlinger TR, Eeckhoute J, Brodsky AS, Keeton EK, Fertuck KC, Hall GF, et al. Genome-wide analysis of estrogen receptor binding sites. Nat Genet. 2006;38:1289-97.

36. Amaravadi $\mathrm{R}$, Kimmelman AC, White $E$. Recent insights into the function of autophagy in cancer. Genes Dev. 2016;30:1913-30.

37. Zeng Q, Liu J, Cao P, Li J, Liu X, Fan X, Liu L, Cheng Y, Xiong W, Li J, et al. Inhibition of REDD1 sensitizes bladder urothelial carcinoma to paclitaxel by inhibiting autophagy. Clin Cancer Res. 2018;24:445-59.

38. Wang L, Yao L, Zheng YZ, Xu Q, Liu XP, Hu X, Wang P, Shao ZM. Expression of autophagy-related proteins ATG5 and FIP200 predicts favorable diseasefree survival in patients with breast cancer. Biochem Biophys Res Commun. 2015;458:816-22.

39. Dong LW, Hou YJ, Tan YX, Tang L, Pan YF, Wang M, Wang HY. Prognostic significance of Beclin 1 in intrahepatic cholangiocellular carcinoma. Autophagy. 2011;7:1222-9.

40. Liu X, Xiao ZD, Han L, Zhang J, Lee SW, Wang W, Lee H, Zhuang L, Chen J, Lin HK, et al. LnCRNA NBR2 engages a metabolic checkpoint by regulating AMPK under energy stress. Nat Cell Biol. 2016;18(4):431-42.

41. Zhu P, Wang Y, Wu J, Huang G, Liu B, Ye B, Du Y, Gao G, Tian Y, He L, Fan Z LncBRM initiates YAP1 signalling activation to drive self-renewal of liver cancer stem cells. Nat Commun. 2016;7:13608.

42. Katz Y, Wang ET, Airoldi EM, Burge CB. Analysis and design of RNA sequencing experiments for identifying isoform regulation. Nat Methods. 2010;7:1009-15.

43. Huelga SC, Vu AQ, Arnold JD, Liang TY, Liu PP, Yan BY, Donohue JP, Shiue L, Hoon S, Brenner S, et al. Integrative genome-wide analysis reveals cooperative regulation of alternative splicing by hnRNP proteins. Cell Rep. 2012;1:167-78.

44. Wigerup C, Pahlman S, Bexell D. Therapeutic targeting of hypoxia and hypoxia-inducible factors in cancer. Pharmacol Ther. 2016;164:152-69.

45. Lyssiotis CA, Kimmelman AC. Metabolic interactions in the tumor microenvironment. Trends Cell Biol. 2017;27:863-75.

46. Choudhry H, Harris AL, Mclntyre A. The tumour hypoxia induced noncoding transcriptome. Mol Asp Med. 2016;47-48:35-53.

47. Carroll JS, Hickey TE, Tarulli GA, Williams M, Tilley WD. Deciphering the divergent roles of progestogens in breast cancer. Nat Rev Cancer. 2017;17:54-64.

48. Bhan A, Hussain I, Ansari KI, Kasiri S, Bashyal A, Mandal SS. Antisense transcript long noncoding RNA (InCRNA) HOTAIR is transcriptionally induced by estradiol. J Mol Biol. 2013;425:3707-22.

49. Ghoussaini M, Fletcher O, Michailidou K, Turnbull C, Schmidt MK, Dicks E, Dennis J, Wang Q, Humphreys MK, Luccarini C, et al. Genome-wide association analysis identifies three new breast cancer susceptibility loci. Nat Genet. 2012:44:312-8. 